\title{
The Effect of Tidal Stripping on Composite Stellar Populations in Dwarf Spheroidal Galaxies
}

\author{
Laura V. Sales, ${ }^{1}$ Amina Helmi, ${ }^{1}$ and Giuseppina Battaglia ${ }^{2}$ \\ ${ }^{1}$ Kapteyn Astronomical Institute, P.O. Box 800, 9747 AD Groningen, The Netherlands \\ ${ }^{2}$ European Organisation for Astronomical Research in the Southern Hemisphere, Karl-Schwarzschild-Straße 2, \\ 85740 Garching bei Muenchen, Germany \\ Correspondence should be addressed to Laura V. Sales, lsales@astro.rug.nl
}

Received 30 April 2009; Revised 30 August 2009; Accepted 1 October 2009

Academic Editor: Andrey V. Kravtsov

Copyright (c) 2010 Laura V. Sales et al. This is an open access article distributed under the Creative Commons Attribution License, which permits unrestricted use, distribution, and reproduction in any medium, provided the original work is properly cited.

\begin{abstract}
We use N-body simulations to study the effects of tides on the kinematical structure of satellite galaxies orbiting a Milky Waylike potential. Here we focus on the evolution of a spherical, dark matter dominated satellite, which is modelled with two stellar components set ab initio to be spatially and kinematically segregated, in a way that resembles the configuration of the metal poor and metal rich stellar populations in several dwarf spheroidals of the Local Group. We find that an important attenuation of the initial differences in the distribution of the two stellar components occurs for orbits with small pericentric radii. This is mainly due to (i) the loss of the gravitational support provided by the dark matter component after tidal stripping takes place and (ii) tides preferentially affect the more extended stellar component, leading to a net decrease in its velocity dispersion as a response for the mass loss, which thus shrinks the kinematical gap. We apply these ideas to the Sculptor and Carina dwarf spheroidals. Differences in their orbits might help to explain why in the former a clear kinematical separation between metal poor and metal rich stars is apparent, while in Carina this segregation is significantly more subtle.
\end{abstract}

\section{Introduction}

Dwarf galaxies are by number the most common kind of galaxies in the Local Group. Among all types of dwarf galaxies, such as dwarf irregulars, dwarf ellipticals, transition types, and dwarf spheroidal (dSphs), the latter dominate the satellite population of the large spirals.

Dwarf spheroidal galaxies are faint (with luminosities between $10^{3}-10^{7} L_{\odot}$ ), metal poor and tend not to be rotationally supported. The dark matter mass enclosed within the optical extent of these small objects ranges between $10^{5}-10^{8} M_{\odot}[1]$, which extrapolated at large radii would give virial masses of the order of $10^{8}-10^{9} M_{\odot}$ and higher [2]. The star formation histories of these small objects are known to be complex and vary from object to object, but all dSphs contain ancient stellar populations, with ages $>10 \mathrm{Gyr}$ old. In most $\mathrm{d}$ Sphs the ancient stellar component is the dominant one or even the only one present (e.g., Sculptor, Draco, Ursa Minor); there are however a few cases where intermediate age stars (3-6 Gyr old) are also present (such as Carina) or dominate the overall stellar population (e.g., Fornax, Leo I) [3-5].

An intriguing observational feature of $\mathrm{dSphs}$ is that, notwithstanding their small sizes, the star formation and chemical enrichment histories are not uniform inside these objects, but varied as exemplified by the presence of spatial gradients in the average metallicity and sometimes age properties of their stellar populations. Harbeck et al. [6] used wide-field imaging to study a set of 9 Local Group dwarfs and examined the spatial distribution of stars in different evolutionary phases as selected from the colour-magnitude diagram (CMD). In particular, the authors used horizontal branch (HB) stars, known to be ancient (>10 Gyr old), and determined the relative spatial distribution of the blue and red (horizontal branch) populations (BHB and $\mathrm{RHB}$, resp.) in order to identify the existence of metallicity/age variations. Harbeck et al. found that segregation of populations may not be uncommon in dSphs although it is not necessarily present in all of them. 
Further new evidence on the presence of multiple populations came a couple of years later, when Tolstoy et al. [7] added to wide-field imaging also the information from wide-field spectroscopy, which yielded metallicities and line-of-sight velocities for hundreds individual stars in the Sculptor dSph. These authors found that metal rich stars in Sculptor are more centrally concentrated and have on average lower velocity dispersions than those of the metal poor component, which on the contrary show hotter kinematics and are more extended. Clear stellar population gradients have been confirmed for Tucana, Sextans, Sculptor and And VI [6], and more recently in Draco and And II $[8,9]$. On the other hand, weak or no gradients have been found in other satellite galaxies of the Local Group as well, such is the case for Carina, Leo I [10], And III and And V-VI [6]. Carina, for example, shows a mild segregation between $\mathrm{HB}$ and red clump stars, comparable in amplitude to the metallicity gradient found for spectroscopically confirmed Carina red giant stars [11]. When kinematical information is also available it is found that these stellar populations differences sometimes clearly show up as multiple kinematical components as in the case of Sculptor and Fornax [12]. In other cases such as in Carina the kinematical distinction of stars with different metallicities is more subtle.

The eventual occurrence and evolution of this spatial and kinematical segregation of stars in dwarf galaxies constitutes an interesting problem for models of galaxy formation. Tolstoy et al. [7] put forward several hypotheses in order to explain their findings in Sculptor: two episodes of star formation with an inactive period between them, the accretion of gas from the dwarf surroundings able to cool and condensate in the core of them or the photoevaporation of the outer gas layers due to a UV background [7]. In the recent work, Kawata et al. [13] followed the formation of a dwarf galaxy in a self-consistent cosmological numerical simulation up to redshift $\sim 6$. These authors found that the complex interplay between star formation and feedback, coupled to chemical evolution, resulted in a system with a marked metallicity gradient. Kawata et al. thus proposed that a sufficiently steep continuous gradient of a single stellar population may appear to observations as two kinematically different metal-poor and metal-rich populations. Even though this constitutes an interesting possibility, the metallicity distribution and velocity dispersion profiles in this model are only marginally consistent with observations, and the system may evolve further (e.g., in mass) from redshift 6 to the present-day.

Tides might also play an important role on the evolution of multiple stellar components in dSphs. Given the proximity to the primary galaxy, tidal effects are likely to be relevant on satellite galaxies such as the MW dSph. In some models, the strong tidal field induced by the proximity to the primary may lead to fundamental changes in the dwarfs internal configuration. These include the development of bars and bending instabilities that strongly affect the internal kinematics of the satellite, transforming rotationally supported systems into hot spheroidals $[14,15]$. Such processes are triggered especially for eccentric orbits, implying that the properties of the dwarfs we observe today around bright galaxies could possibly give us indirect clues about their primordial (original) state, that is, before interactions with the primary shaped them differently.

In this paper we explore how gravitational effects may induce a mixing of two initially distinct stellar components in dwarf spheroidal galaxies orbiting an MW-like host potential. Our aim is to gauge the evolution of multiple component dwarfs as they move through the host potential and how this depends on their specific orbital path. In particular, we focus on the differences shown when the same fiducial satellite is placed and evolved on four orbits around the host with different periods and pericenter distances. We therefore do not attempt to explain the origin of such metallicity/kinematical segregation in the dwarfs in the present paper.

We describe the numerical experiments in Section 2, present and discuss our results in Section 3, and summarise our findings in Section 5.

\section{Numerical Experiments}

Observations of the radial velocities of stars in dSph suggest that these are in general dark matter dominated systems, with only a very small $(\leq 5 \%)$ fraction of their baryonic mass in gaseous form [3-5]. This allows a reasonable modeling of their present-day properties and recent evolution by means of collisionless N-body simulations. Here we use GADGET2 [16] to simulate satellite galaxies orbiting in a (static) Milky Way-like halo. Specifically, we focus on the evolution of satellites with two spherical stellar components embedded in an extended dark matter halo.

2.1. The Model for the Host Potential. We model the (Milky Way) host potential as a (fixed) three-component system with the following:

(i) a Navarro et al. [17] dark matter halo:

$$
\rho(r) \propto \frac{1}{x(1+c x)^{2}}
$$

with mass $M_{\mathrm{dm}}=1 \times 10^{12} M_{\odot}$ and concentration $c=$ 12 (Klypin et al. [18]),

(ii) a Hernquist [19] bulge:

$$
\rho(r)=\frac{M_{\mathrm{blg}} d}{2 \pi r} \frac{1}{(r+d)^{3}}
$$

with mass and scale length: $M_{\mathrm{blg}}=3.4 \times 10^{10} M_{\odot}$ and $d=0.7 \mathrm{kpc}$,

(iii) a Miyamoto and Nagai [20] disk:

$$
\rho(R, z)=\frac{b^{2} M_{\mathrm{dsk}}}{2 \pi} \frac{a R^{2}+\left(a+3 \sqrt{z^{2}+b^{2}}\right)\left(a+\sqrt{z^{2}+b^{2}}\right)^{2}}{\left[R^{2}+\left(a+\sqrt{z^{2}+b^{2}}\right)^{2}\right]^{5 / 2}\left(z^{2}+b^{2}\right)^{3 / 2}}
$$

with parameters: $M_{\mathrm{dsk}}=1 \times 10^{11} M_{\odot}, a=6.5 \mathrm{kpc}$ and $b=0.26 \mathrm{kpc}$ (Johnston et al. [21]). 
2.2. The Model for the Satellite. The satellite model consists of two baryonic components following Plummer density profiles:

$$
\rho_{i}=\frac{3 M_{i} b_{i}^{2}}{4 \pi} \frac{1}{\left(r^{2}+b_{i}^{2}\right)^{5 / 2}}
$$

of different masses $M_{i}$ and scale lengths $b_{i}$ that mimic a concentrated metal rich and more extended metal poor stellar components $\left(C_{1}\right.$ and $C_{2}$, resp.). Both spheres are embedded within a Hernquist-profile dark matter halo. The masses of each of these components are set to $1.5,3.5 \times 10^{6}$ and $2 \times 10^{9} M_{\odot}$ for $C_{1}, C_{2}$, and dark halo. The Plummer spheres scale lengths for $C_{1}$ and $C_{2}$ are $b_{1}=0.11 \mathrm{kpc}$ and $b_{2}=0.35 \mathrm{kpc}$, respectively. For the dark matter, the scale radius is $a=2.7 \mathrm{kpc}$, which gives a half mass radius $\sim 6.5 \mathrm{kpc}$.

The satellite galaxy is modelled using $\sim 310000$ particles distributed in a dark matter halo (200 000) and two luminous components (110000) set by construction to have an initial spatial and kinematic segregation. The procedure to generate the initial condition for simulations of compound galaxies was developed by Hernquist [22] and invokes the moments of the Collisional Boltzman Equations (CBEs) to self-consistently approximate the (unknown) distribution functions of particles in all galaxy components. We briefly describe this procedure in what follows and refer the reader to the original paper [22] for further details.

For an isotropic, spherically symmetric system, the second moment of the CBE can be written as

$$
\left\langle v_{r}^{2}\right\rangle=\frac{1}{\rho} \int_{r}^{\infty} \rho(r) \frac{d \Phi}{d r} d r,
$$

where $\left\langle v_{r}^{2}\right\rangle$ is the velocity dispersion in the radial direction, $\rho$ is the mass density distribution, and $\Phi$ the total gravitational potential of the system. This formula can be extended to the case where several subcomponents coexist in mutual equilibrium. For such configurations, the second velocity moment of the component $C_{j}$ would be given by

$$
\left\langle v_{r, j}^{2}\right\rangle=\frac{1}{\rho_{j}} \int_{r}^{\infty} \sum_{i=1}^{n_{c}} \rho_{j} \frac{d \Phi_{i}}{d r} d r
$$

(where $j$ and $i$ vary over the $n_{c}$ different components of the system). Once the density profiles $\rho_{j}$ for each of the components have been fixed, the solution to this equation may be found, either analytic or numerically. To set up the particles velocities we assume isotropic Gaussian distribution functions. This procedure then allows us to assign a velocity $v$ to a particle located at a distance $r$ in component $C_{j}$, where $v$ is taken randomly from the distribution:

$$
F_{j}(r, v)=4 \pi\left(\frac{1}{2 \pi \sigma_{j}^{2}}\right)^{3 / 2} v^{2} \exp \left(-\frac{v^{2}}{2 \sigma_{j}^{2}}\right)
$$

with dispersion $\sigma_{j}=\left\langle v_{r, j}^{2}\right\rangle$.

The softening lengths in multiple component systems must be chosen carefully. We follow the prescriptions described in [23], which give in each case is: $\epsilon_{C 1} \sim 7 \mathrm{pc}$,
$\epsilon_{\mathrm{C} 2} \sim 21 \mathrm{pc}$, and $\epsilon_{\mathrm{DM}} \sim 60 \mathrm{pc}$. Figure 1 shows the resulting (projected) density and velocity profiles for this model (hereafter, $R$ and $r$ refer to projected and three-dimensional distances, resp.). As a consequence of this set-up, the initial configuration of the simulated dwarf has a slightly rising $\sigma_{\text {los }}$ profile in the inner regions, that can be explained as the transition from the more concentrated to the more extended stellar components; [24].

The baryonic components of the simulated dwarf are deeply embedded within the potential well of the dark matter halo. The core radius is $\sim 7.3 \% r_{\max }$, where $r_{\max }$ is the radius at which the circular velocity of the dark matter halo peaks; in good agreement with suggestions from numerous theoretical models [25-27]. Such segregation with respect to the dark matter halo turns the stellar components of the satellite galaxies more resilient to tidal stripping, increasing the probability of survival for many orbital times without appreciable changes in their observable properties. Notice that stars account only for less than $0.25 \%$ of the total mass, the bulk of the dwarf mass is largely dominated by the dark matter component, with an M/L $\sim 35$ within the tidal radius (we have assumed here a conversion factor $\gamma=2.9$ to compute the luminosity out of the mass in the baryonic components, this is roughly consistent with a $\sim 10 \mathrm{Gyr}$ old stellar population of mean metallicity $[\mathrm{Fe} / \mathrm{H}]=-2$ (as derived from the BASTI model isochrones) (http://www.te.astro.it/BASTI/index.php.).

The stellar and dark matter masses have been chosen to approximately match the typical line-of-sight velocities dispersions $\left(\sigma_{\text {los }} \sim 10 \mathrm{~km} / \mathrm{s}\right)$, luminosities and structural parameters (such as core $R_{c}$ and tidal $R_{t}$ radius) that are observed in Local Group satellites. Throughout this paper we define the characteristic radii $R_{c}$ and $R_{t}$ by finding the bestfitting King profiles to the projected density of particles in the luminous components. Notice that with this definition, the tidal radius is not necessarily related to the physical radius that divides the bound from the unbound population of stars in cases where tidal stripping takes place.

The simulated satellite constructed in this way is first relaxed (evolved in isolation) during $1 \mathrm{Gyr}$ before being placed on orbit around the host potential for a longer timescale of $t \sim 6 \mathrm{Gyr}$.

The stability of our initial conditions against numerical and relaxation effects can be appreciated in Figure 1. Overplayed using thin lines we show the final configuration (projected density and velocity dispersion profiles) for the same satellite model but evolved in isolation during $t \sim 6$ Gyr. The close match between the initial conditions (thick lines) and this control model (thin lines) indicates that any departure from the initial set up will be driven by the evolution of the satellite into the host potential and is not a consequence of an out-of-equilibrium initial configuration.

2.3. Satellite Orbits. The gravitational forces that a satellite galaxy experiences are strongly dependent on its orbit, in particular on the pericenter distances and the orbital radial period (that determine the number of pericenter passages that occur in a given time span), both of which are related 


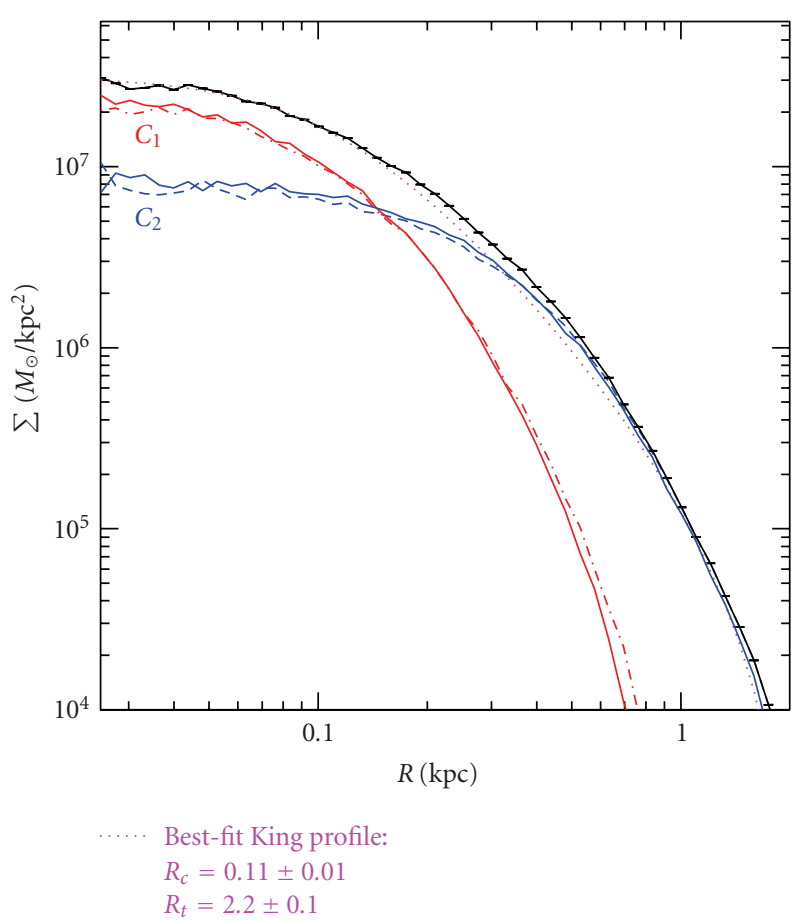

(a)

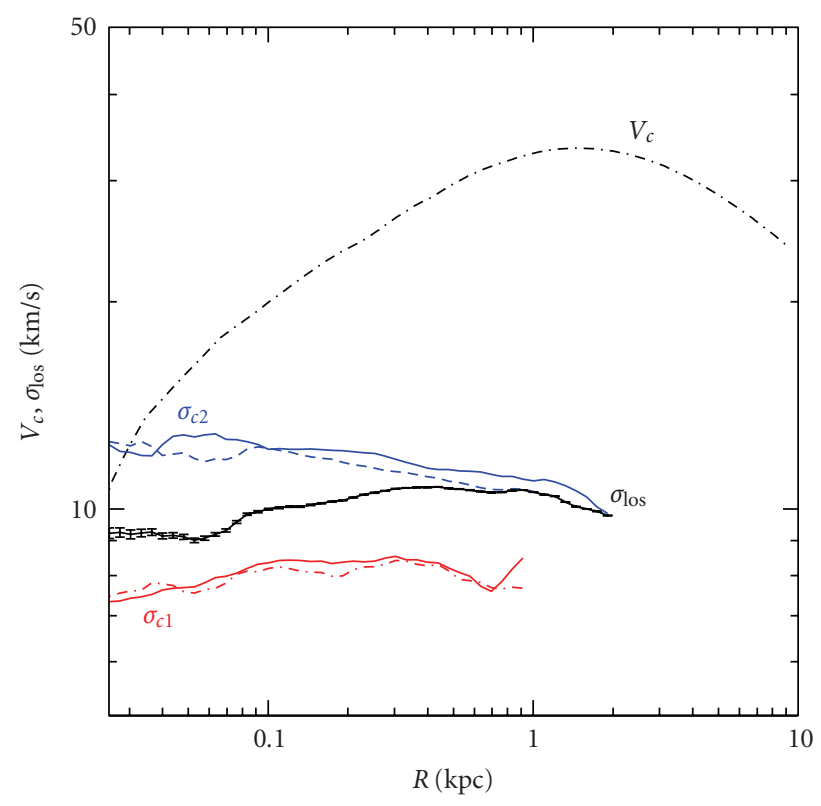

(b)

FIGURE 1: Surface mass density (a) and velocity (b) profiles for the simulated satellite as a function of the (projected) distance from the centre, $R$. (a), (b), red dot-dashed and blue-dashed curves distinguish the contributions from the more concentrated $\left(C_{1}\right)$ and more extended $\left(C_{2}\right)$ components, respectively, while the thick solid black shows the total values obtained for all the stars (i.e., no distinction between populations). In the left panel, the magenta dotted line shows the best-fit King profile obtained for the total distribution of stellar mass, and the numbers quoted correspond to the derived core and tidal radii, respectively. The right panel shows the circular velocity (black dashed) plus the line-ofsight velocity dispersion profiles associated to each luminous component. Poisson uncertainties are shown for the total density and velocity dispersion profiles in our model, although they are generally smaller than the thickness of the curves. Thin lines in (a), (b) correspond to a control model, which is evolved from this initial configuration in isolation during $t \sim 6$ Gyr.

to the eccentricity of the orbit. While the dark matter halo associated to a satellite provides some sort of "shielding" for the baryons, the removal of the luminous component may start after the dark mass has been reduced to less than $10 \%$ its original value. However, the changes in the equilibrium state produced by a significant depletion of the dark matter halo could in principle affect the properties of the baryonic component even before begin to be stripped.

We explore this by placing our fiducial satellite in four different orbits within the host potential (see Figure 2), and integrating its evolution in time for $t \sim 6 \mathrm{Gyr}$ (this may vary $\pm 0.3 \mathrm{Gyr}$ depending on the timing of their orbit apocenter passage). This integration timescale is chosen to be consistent with the average time of accretion of the surviving population of satellites found in semianalytical models and numerical simulations of galaxy formation [3033]. In order to facilitate the interpretation of the results in terms of Local Group galaxies, we have generated two orbits from the present day positions, radial velocities and proper motions of Sculptor and Carina, as reported by Piatek et al. $[28,29]$. These correspond to Orb1 and Orb2 in Figure 2, for Sculptor and Carina respectively. Even though the errors in the velocity determinations are appreciable $(\sim 50 \%)$, which leads to a wide range of possible orbits, the more likely values give rise to interesting differences in their orbits (Orb1 and Orb2). Orbits 3 and 4 correspond to two random orbits generated under the condition of comparable present-day galactocentric distances $(r \sim 90-100 \mathrm{kpc})$ to orbits 1 and 2 .

Figure 2 shows that despite their similar present-day distance from the host, an orbit such as that suggested by Sculptor's proper motions (Orb1) is significantly more external than Carina's (Orb2), with apocenters exceeding $150 \mathrm{kpc}$ and pericenter distances of $\sim 75 \mathrm{kpc}$. Orb2 is much more constrained to the inner parts of the Milky Way potential, with pericenters smaller than $30 \mathrm{kpc}$ and orbital periods $\sim 1.6 \mathrm{Gyr}$, almost 2.4 times shorter than estimated for Scl. Orb3 has comparable pericenters to Orb2, but its apocenters are $50 \%$ larger, which translates into a longer orbital radial period. Finally Orb4 is similar to Orb2, and is also consistent with Car proper motions, but has much smaller pericenter values $\left(r_{\text {per }} \sim 9 \mathrm{kpc}\right)$.

A given satellite placed on these four orbits will thus be subject to different tidal field strengths, and consequently, will present structural and dynamical properties that may differ substantially after $t \sim 6 \mathrm{Gyr}$ of evolution in the host potential. We focus on these aspects in the next section, with special attention to the effects imprinted in the internal kinematics of the stellar components. At this 


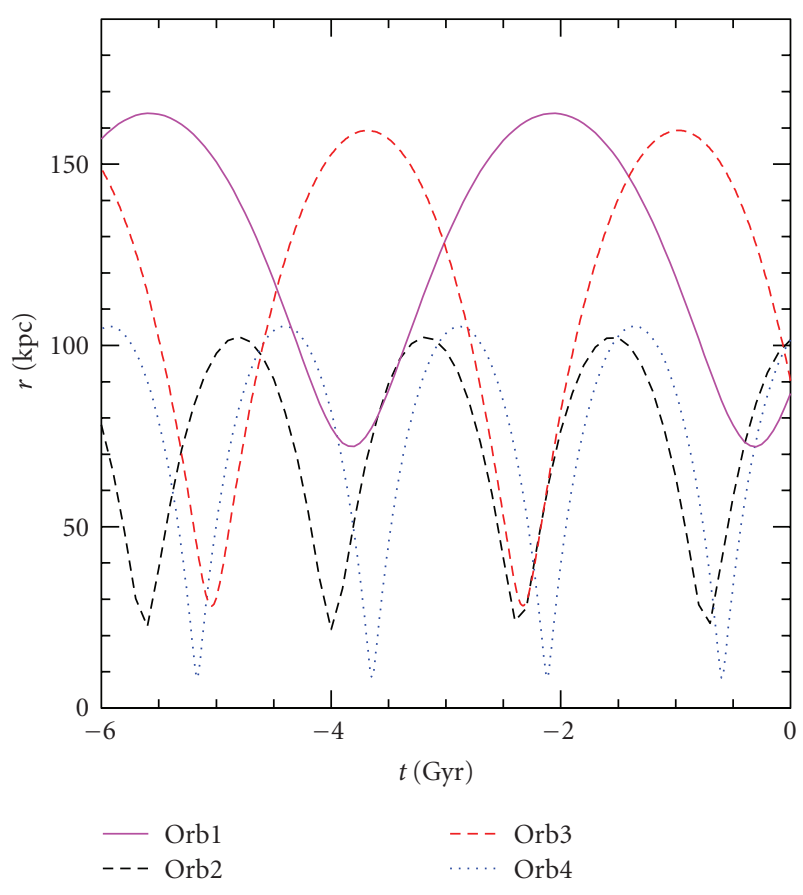

FIGURE 2: Galactocentric distance $r$ as function of time for the four different orbits explored in this paper. Orbits 1 and 2 have been generated from the present-day positions and proper motions of the Sculptor and Carina dwarfs, as reported by Piatek et al. [28, 29] respectively.

point in time, the satellite is located near the apocenter for Orb2 and Orb4, where it will spend most of its time. The system will then reconfigure to its new equilibrium state and the configuration during apocenter passages is the most representative of their final state. On the other hand, at the final snapshot, satellites in Orb1 and Orb3 are at arbitrary points on their orbits. However, given their more external orbital configuration, the final properties for these systems do not strongly depend on the location along their orbits.

\section{Results}

After $\sim 6 \mathrm{Gyr}$ of evolution of the model satellite in the host potential, we are able to identify a self-bound satellite remnant for all four explored orbits. However, in spite of the originally identical settings, the structural properties of the surviving dwarf vary appreciably depending on the different orbits. A brief summary of the retained mass fractions and the structural parameter for each model can be found in Table 1.

3.1. Final Distribution of Satellite's Stars. Figure 3 shows a snapshot view of the final system evolved in each of the orbits. Black, blue and red correspond to the dark matter, metal poor and metal rich stellar components, respectively. Visual inspection of this figure suggests that the shapes of the remnants at the final time keep some imprints about the history of each dwarf. For example, for external orbits such as
Orb1 and Orb3, the stars in both components are distributed in a spherical fashion, reminiscent of (and determined by) the initial setting of the progenitor. On the other hand, satellites on Orb2 and Orb4 have developed tails of unbound material as a result of a stronger tides suffered during their small pericenter passages. It is interesting to note that since tidal disruption affects earlier and more appreciably the extended $C_{2}$ component than $C_{1}$, the shape of the metal poor stellar distribution may be more elongated than the metal rich when mass depletion has not yet proceeded into the inner regions of the dwarf. Such is the case of the model in Orb2, which has retained $100 \%$ and $88 \%$ of the initial mass in components $C_{1}$ and $C_{2}$, respectively (see Table 1). However, once tidal stripping affects also the more concentrated population, the distribution of metal rich and metal poor stars will both turn elliptical in shape as seen for Orb4.

The final projected mass density profiles for each simulation are shown in Figure 4, together with their bestfitting King profiles. The corresponding core and tidal radii ( $R_{c}$ and $R_{t}$ resp.) are listed in Table 1 , together with the fractions of mass in each component that has remained bound. We have tested the effect of the particular alignment of the tidal tails with the line-of-sight to the observer by computing the density profiles obtained from 10 different random projections of each satellite. These are depicted by thin lines in Figure 4, which show that orientation is not particularly important for the fairly undisturbed systems, and its impact is minor in the case of Orb4.

As quoted in Table 1, the tidal forces have affected significantly less the stellar components than the associated dark matter halo of the dwarf. For example, the satellite on orbits 2 and 3 has retained only 5\% and $20 \%$ of its dark matter mass, respectively, but more than $90 \%$ of the initial stellar mass. Notice that the structural parameters of the baryons (quantified by the projected King profile best fit parameters) are robust to the ongoing pruning of the satellite's outskirts, in particular, the core radii show no variations in any of the experiments (see discussion in [34]). On the other hand, the external parts, and consequently the tidal radius do show more significant changes. In the case of the orbit with the smallest pericenters (Orb4) the tidal radius has shrunk by a factor $\sim 2$ from its original value. Also projection effects are in this case more important, due to different contributions of unbound stars according to the alignment of the tails with the lineof-sight [35]. For the other three orbits, the tidal radius and density profiles have been only barely affected by the stripping.

We observe that the segregation in the spatial distribution between components $C_{1}$ and $C_{2}$ is also modified, although remains present in all of our experiments (however, this clearly depends on the length of the integration, see Section 3.3). For example, the mass ratio between $C_{1}$ and $C_{2}$ within the core radius tends to increase in cases where tidal stripping is significant. This is naturally expected as tides will lead to a larger (and earlier) stripping of the most extended $\left(C_{2}\right)$ component compared to $C_{1}$. For orbits 2 and 4, $m_{C_{1}} / m_{C_{2}}$ increases from $\sim 2$ in the initial model, to 2.6 and 


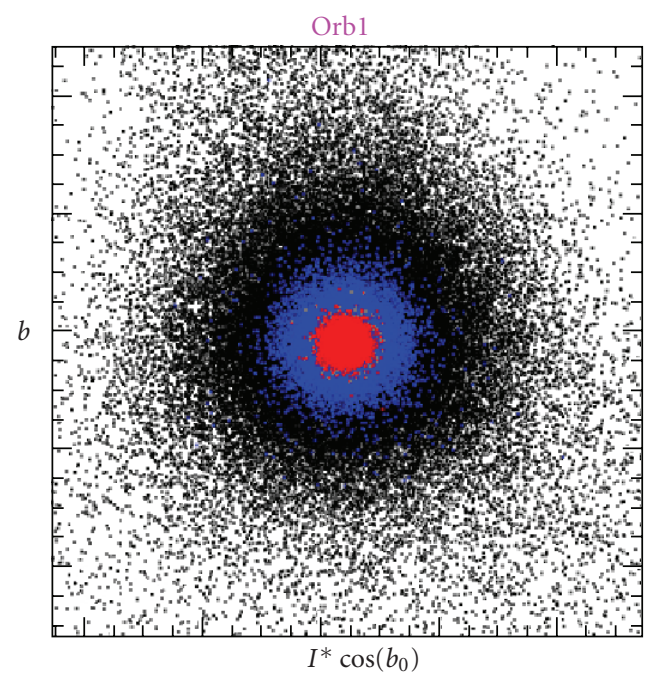

(a)

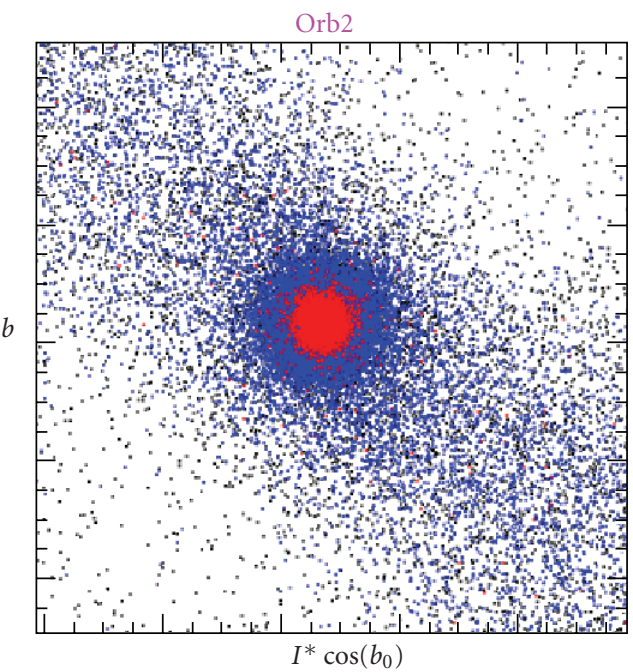

(c)

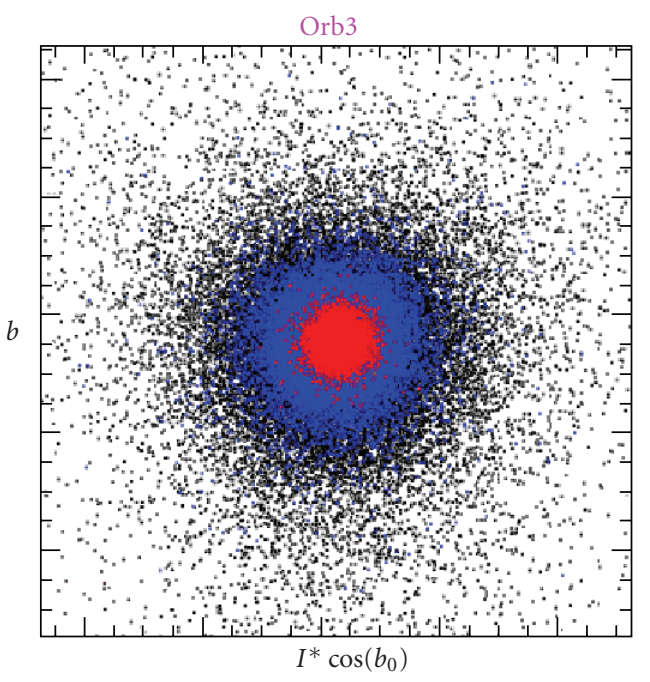

(b)

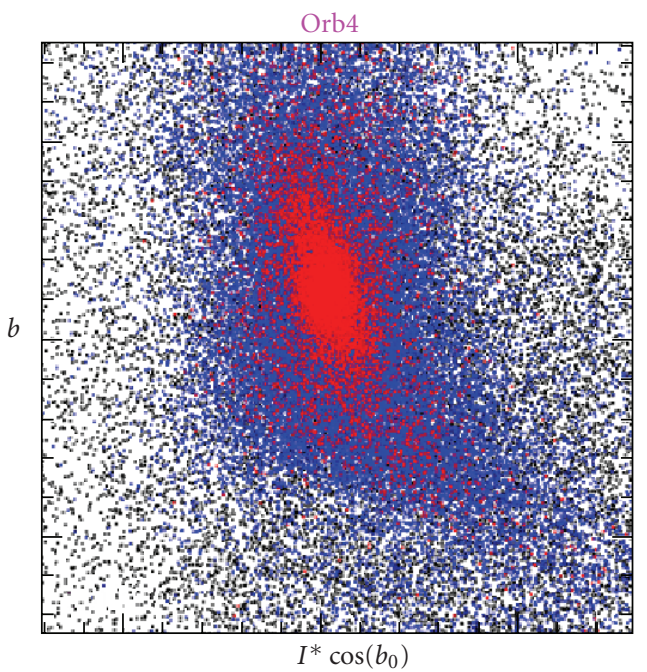

(d)

FIGURE 3: Final projected view (arbitrary units) of the dark matter (black), metal poor (blue) and metal rich (red) stellar components for the models evolved in orbits 1-4 during $t \sim 6$ Gyr. Due to the different degree of tidal disruption experienced in each case, some satellites have turned more elliptical in shape in either one (Orb2) or both (Orb4) their stellar components. Satellites in orbits 1 and 4 remain still quite spherical as the progenitor model.

TABLE 1: Structural properties of the satellite models after its evolution for $t=6( \pm 0.3)$ Gyr within the fixed Galactic potential. The first row in the table shows the initial values and subsequent rows show the present-day values for the experiments on Orb1-Orb4. $R_{c}$ and $R_{t}$ refer to the best-fitting King profile values to the surface density profile of the luminous components. The last 3 columns indicate the fraction of mass in each component that remains bound to the dwarf in the final configuration.

\begin{tabular}{|c|c|c|c|c|c|}
\hline Orbit & $R_{c}(\mathrm{kpc})$ & $R_{t}(\mathrm{kpc})$ & $f_{C 1}(\%)$ & $f_{C 2}(\%)$ & $f_{\mathrm{DM}}(\%)$ \\
\hline Initial & $0.11 \pm 0.01$ & $2.2 \pm 0.1$ & 100 & 100 & 100 \\
\hline Orb1 & $0.11 \pm 0.01$ & $2.0 \pm 0.1$ & 100 & 99 & 44 \\
\hline Orb2 & $0.11 \pm 0.01$ & $1.9 \pm 0.1$ & 100 & 88 & 5 \\
\hline Orb3 & $0.11 \pm 0.01$ & $2.2 \pm 0.1$ & 100 & 98 & 20 \\
\hline Orb4 & $0.11 \pm 0.01$ & $1.0 \pm 0.1$ & 87 & 20 & 3 \\
\hline
\end{tabular}




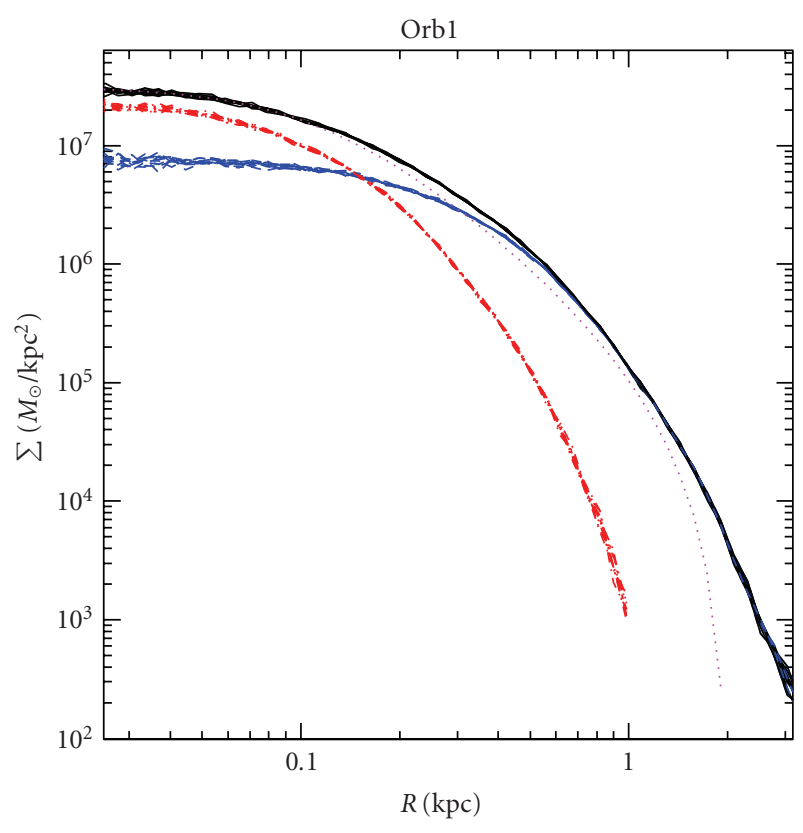

(a)

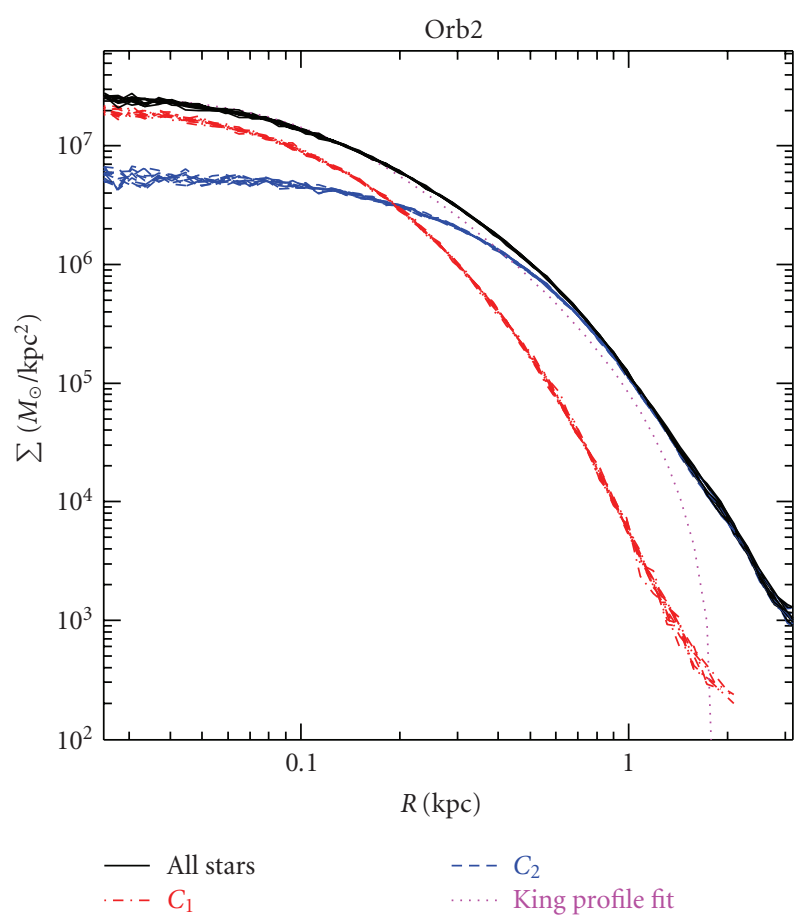

(c)

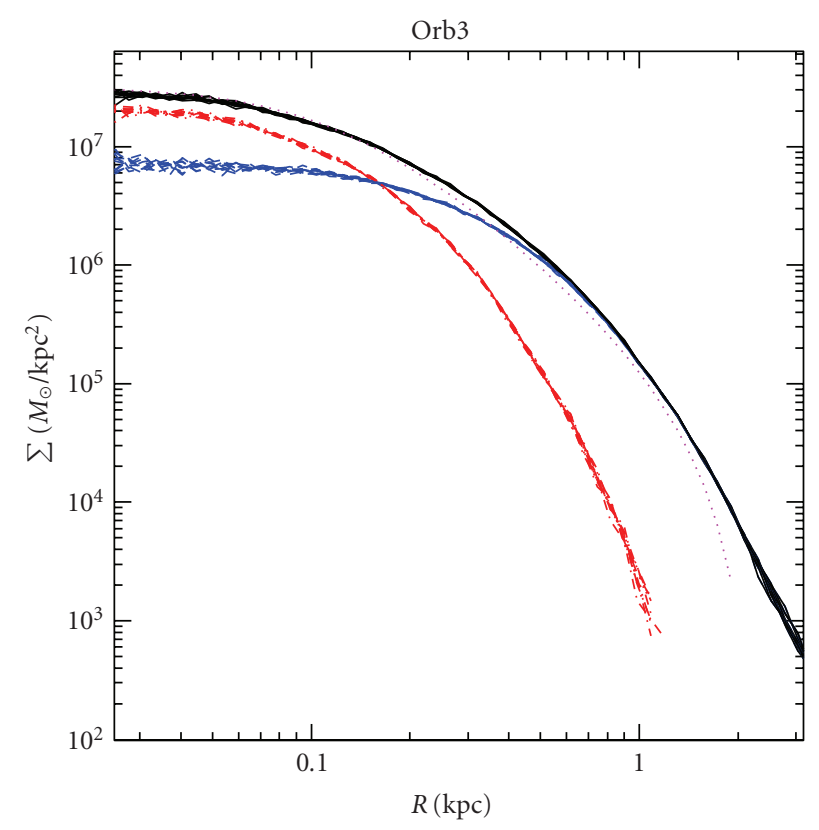

(b)

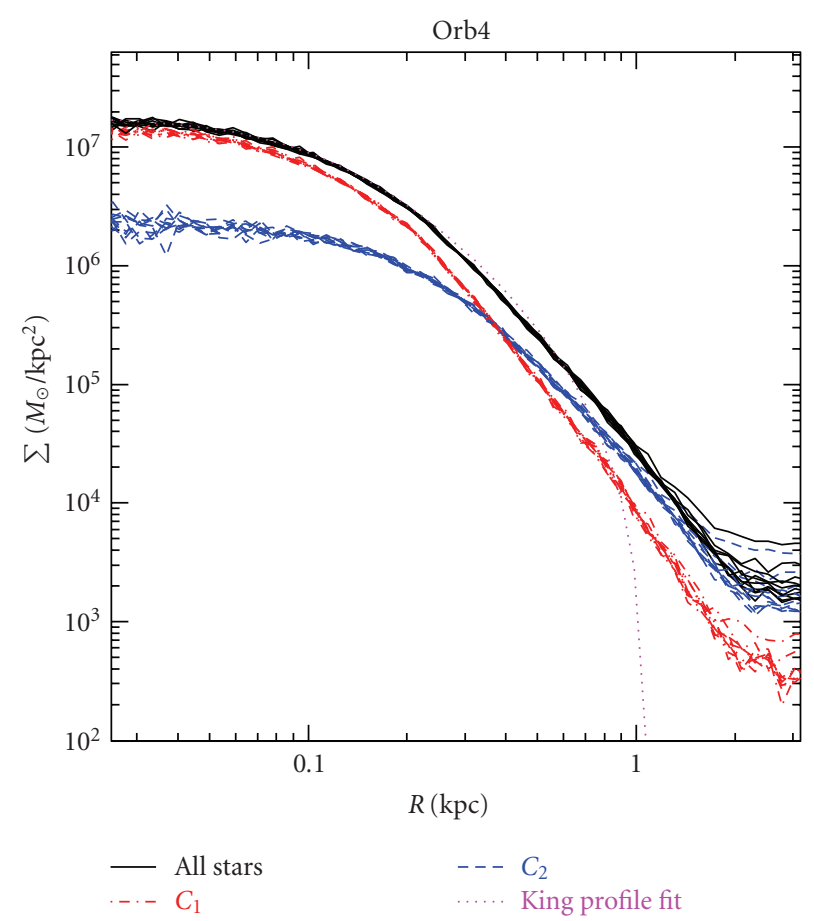

(d)

FIgURE 4: Surface mass density profiles of all our models after $\sim 6 \mathrm{Gyr}$ of integration in the host potential. The color codding is the same as that used in Figure 1.

4.9 , respectively (orbits 1 and 3 show no variation). For the same orbits ( 2 and 4 ) this ratio for the more external region $0.5<R<1 \mathrm{kpc}$ changes from an initial value $m_{C_{1}} / m_{C_{2}} \sim$ 0.02 , to $\sim 0.12$ and $\sim 0.62$, respectively.

This gradient of $m_{C_{1}} / m_{C_{2}}$ with radius implies that the spatial distinction between both luminous components is still present in the dwarf galaxy. Notice however that some redistribution of the stars in the components has taken place. For example, in the external regions $(0.5<R<1 \mathrm{kpc})$ of the dwarfs in orbits 2 and 4, the contribution of particles from component $C_{1}$ changes from negligible (less than $\sim 2 \%$ ) to significant $(\sim 10-60 \%)$ as a result of the ongoing tidal perturbations that require the dwarf to find new equilibrium configurations (see also Figure 3). 
3.2. Dynamics of Satellite's Stars. Also worth highlighting is the dynamical response of the system to such structural changes. The velocity dispersion in spherically symmetric systems is determined by the amount of mass contained within a given radius (i.e., the density profile) and by the velocity anisotropy associated to the particles orbits [38]. We naively expect from the results discussed above that not only the structural parameters but also the kinematical properties (such as the line-of-sight velocity dispersion, $\sigma_{\text {los }}$ ) will be affected by the mass removal experienced by the satellite. This is shown in Figure 5, where we plot the (1D, line-of-sight) velocity dispersion profiles as a function of the projected radius from the center of the satellite. To avoid contamination by unbound particles, and following the method applied to observations [36], we have removed all particles whose line-of-sight velocity exceeds by $\pm 3 \sigma_{\text {los }}$ the mean velocity of the system $\left(\left|\bar{V}(R)-V_{\text {los }}\right|>3 \sigma_{\text {los }}\right)$.

The panels in Figure 5 correspond to each one of the orbits explored, with thin lines showing the velocity dispersion in each radial bin for each population. The long dashed blue and the solid red curves refer to the more extended $\left(C_{2}\right)$ and concentrated $\left(C_{1}\right)$ luminous components, respectively. In order to illustrate the effects introduced by the geometry of the problem and the different alignments between possible streams and the line-of-sight direction we show curves corresponding to 10 different random projections for each satellite. The error bars in this figure are estimated as follows. We randomly extract a (sub)set of 500 particles from the luminous components of the simulated satellite, maintaining the mass ratio between population $C_{1}$ and $C_{2}$ and their spatial distribution. The size of each subsample (500 points) is comparable to the size of current observational samples of line-of-sight velocities in dSphs $[1,36]$. For each subsample we derive $\sigma_{\text {los }}-R$ curves, similar to those shown in Figure 5, albeit with larger noise due to the poorer number statistics. The average scatter between these $\sigma_{\text {los }}-R$ relations obtained from the 100 realizations is then shown as error bars in Figure 5. In order to guide the eye, we have included as well in this figure the initial conditions for the satellite (dotted lines).

Figure 5 shows that when a strong segregation exists between the luminous and dark components, the velocity dispersions of the stars are only weakly affected by the removal of the extended dark halo. For example, experiments Orb1 and Orb3 show that the dark matter halo has been reduced to only $\sim 45 \%$ and $20 \%$, respectively, of the initial mass, yet the kinematics of the luminous components remained essentially intact. Tidal effects proceed outside-in, preserving the central regions of the satellites (where baryons are located) roughly unchanged.

However, the situation is different when trimming sets in the stellar components, as is the case for the satellite on orbits 2 and 4 (Figure 5(e), 5(f)). The tidal stripping tends to shrink the initial gap between the velocity dispersion of the luminous components. Furthermore, the limited sizes of available observational samples ( $\sim 500$ member stars $)$ will lead to additional uncertainties preventing a clear distinction between the kinematics of the two populations, (see [39]). Even though components $C_{1}$ and $C_{2}$ experience a decrease in their $\sigma_{\text {los }}$, they are not affected in the same degree, mainly due to their relative spatial segregation that determines an earlier and stronger removal of stars from component $C_{2}$ compared to $C_{1}$. In such cases, the preferential stripping of the more extended component (mimicking the metalpoor component of satellites) should lead to the presence of metallicity gradients along the stellar streams of the remnant. Evidence for such trends has recently been found in the streams of the Sagittarius dwarf, where BHB are significantly (at $\sim 4.8 \sigma$ level) more abundant than red clump stars in comparison to the core of this galaxy [40]. This is interpreted as the preferential removal of the old metal poor (BHB) stars from the outskirts of Sagittarius, compared to the intermediate-age red clump population that has remained in the core of the dwarf less affected by external tides, in strong analogy with our simulations.

The velocity dispersion profiles for each of the luminous components decline with radius (instead of being roughly flat as in the initial model) as stripping proceeds on the simulated galaxies. Beyond the tidal radius (recall this is defined purely by the best-fitting King profile to the projected mass distribution of the stars), the velocity dispersion increases reaching $\sigma \geq 15 \mathrm{~km} / \mathrm{s}$, as a result of the larger contribution from gravitationally unbound stars (that the $\pm 3 \sigma$ cut described above is unable to remove). This effect is particularly important for Orb2 and Orb4, where the tidal stripping of stars proceeded. Note that this upturn in $\sigma_{\text {los }}$ disappears once the analysis is restricted to particles that remain gravitationally bound to the satellite. In agreement with Klimentowski et al. [35], we find that depending on the orientation of such tails with respect to the line-of-sight, different $\sigma_{\text {los }}$ profiles are obtained. When the radial vector to the satellite is well aligned with the direction of these tails, the contamination by unbound material is maximized, producing a raising velocity dispersion profile towards the dwarf's outskirts.

Taken at face value, these velocity dispersion profiles may seem at odds with the typical flatness of the $\sigma_{\text {los }}$ profiles observed for Local Group dwarfs. As an example, observed data corresponding to the line-of-sight velocity dispersion inferred for Sculptor and Carina are shown in Figures 5(e), 5(f). Data for these two dwarfs was taken from [11, 36, 37], respectively. However, unless the stripping is considerably large, our simulations do still reproduce reasonably flat (or even rising) total velocity dispersion profiles, when no distinction between stars from population $C_{1}$ and $C_{2}$ is made, (see also [24]). This is shown by the magenta, black, red and blue lines in Figures 5(e), 5(f), corresponding to the total velocity dispersion profiles predicted for Orb1-Orb4 (notice color-codding follows Figure 2). Our models in Orb1 and Orb2 reasonably fit the observed velocity dispersion data for Sculptor and Carina, respectively.

We find that while projections have profound effects on $\sigma_{\text {los }}$, it only causes mild variations on the projected density profiles around the formal tidal radius (see Figure 1). A closer look at this indicates that while only $\sim 5-10 \%$ contamination from unbound stars is enough to increase the velocity dispersion profiles by few $\mathrm{km} / \mathrm{s}$, at least twice this fraction is needed to drive significant changes on the surface 


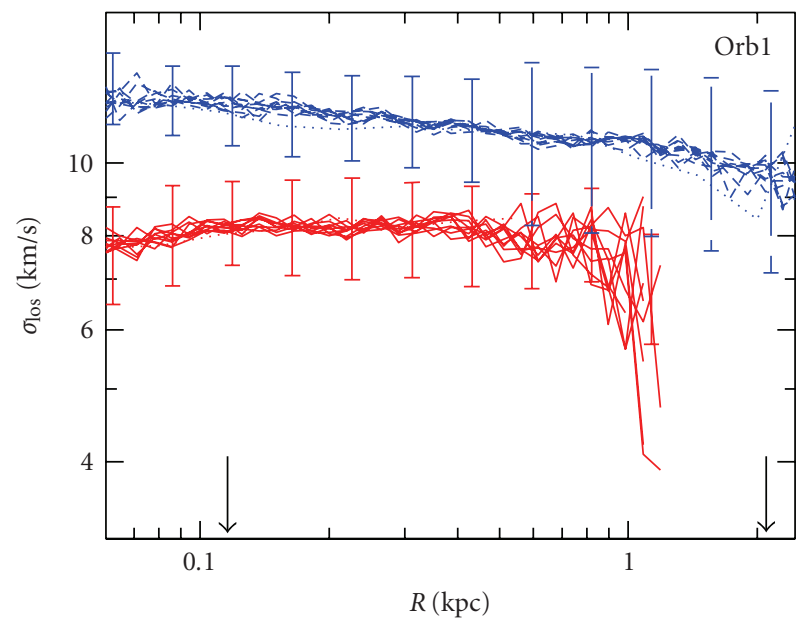

(a)

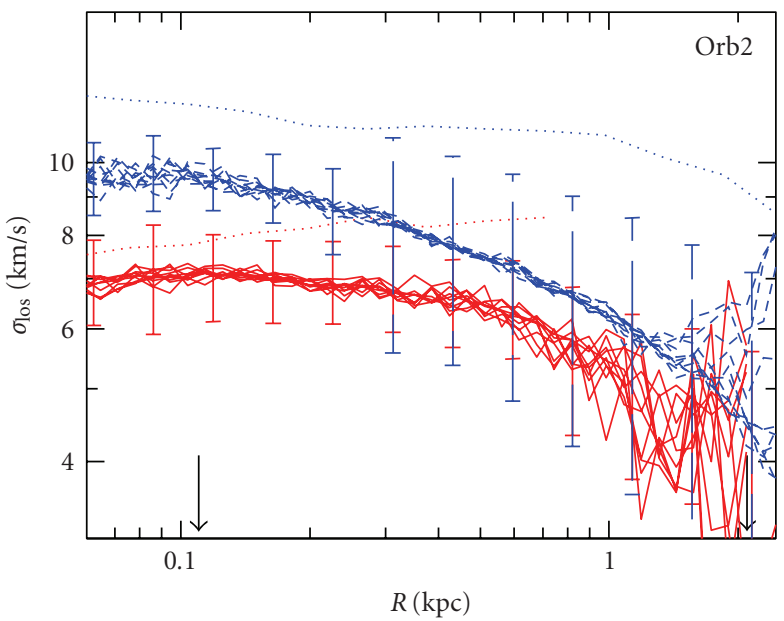

(c)

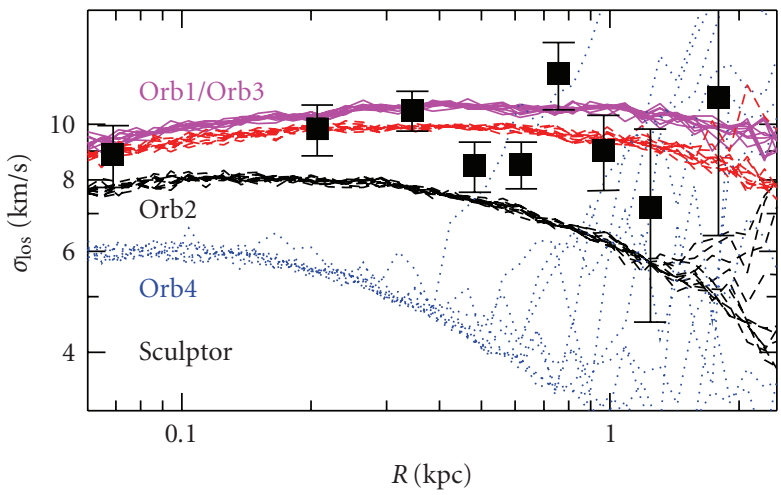

(e)

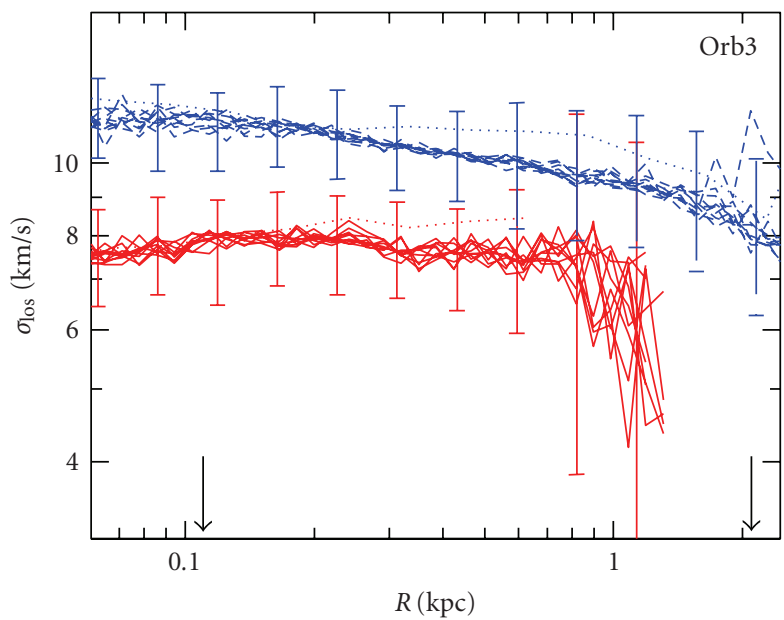

(b)

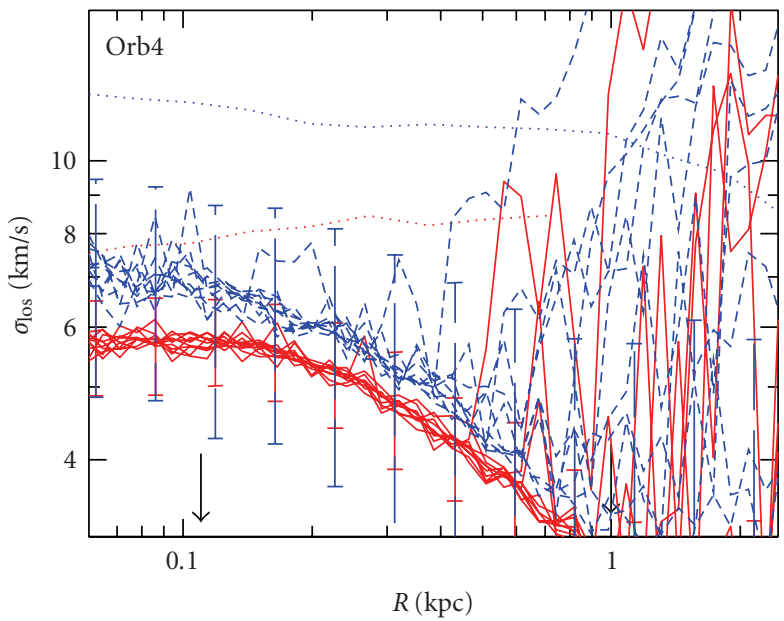

(d)

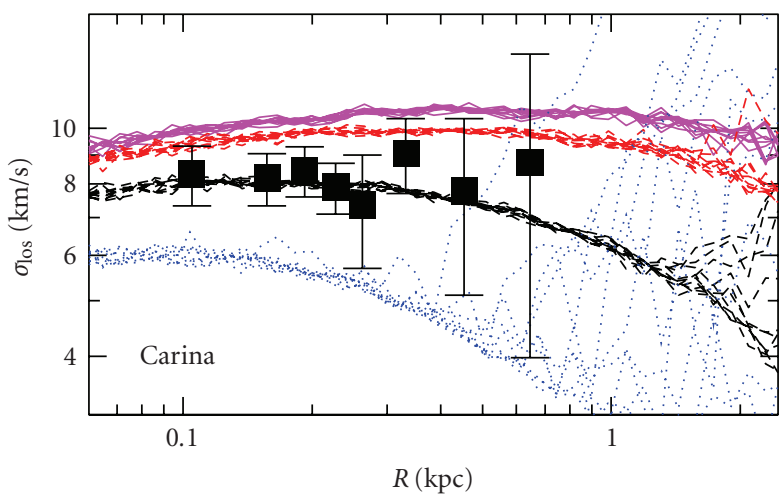

(f)

FIGURE 5: (a)-(d) Line-of-sight velocity dispersions for both luminous components as a function of the (projected) distance from the center of the satellite. Solid red and dashed blue curves correspond to the concentrated $\left(C_{1}\right)$ and the more extended $\left(C_{2}\right)$ components, respectively. The error bars indicate the rms dispersion on the $\sigma_{\text {los }}$ profiles of 100 random samples containing 500 particles each (see text for details). For reference, the dotted lines show the initial configuration of the model satellite. Black arrows indicate the positions of the core and tidal radii in each experiment. (e), (f) Velocity dispersion profiles for Sculptor and Carina dwarfs (squares with error bars). Data was taken from Battaglia et al., koch et al., and Helmi et al. $[11,36,37]$. The total (no distinction between components) $\sigma_{\text {los }}$ profiles for our models in the 10 different projections are also shown in magenta, black, red and blue lines for Orb1-Orb4, respectively. 


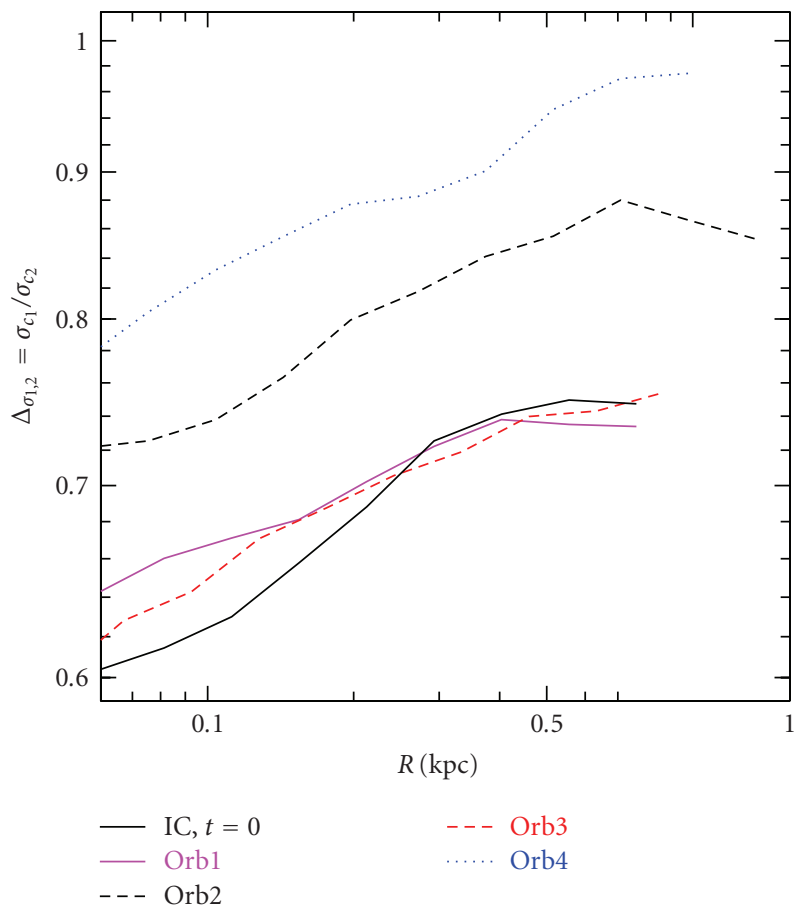

(a)
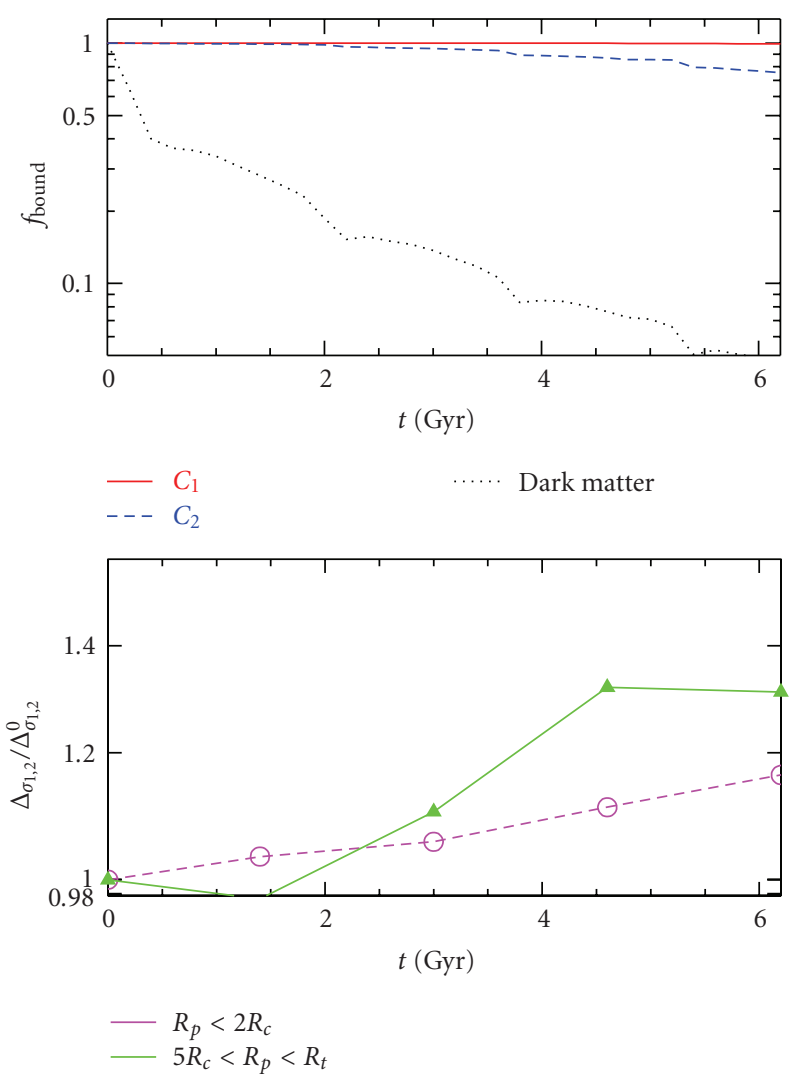

(b)

Figure 6: (a) Ratio between the line-of-sight velocity dispersions for component $C_{1}$ and $C_{2}\left(\Delta_{\sigma_{1,2}}=\sigma_{c 1} / \sigma_{c 2}\right)$ as a function of the projected distance from the center of the dwarf. The mos distant bin considered has at least 20 particles from each component (and more for for bins closer in). (b) The upper panel shows the fraction of mass (compared to the initial value) that remains bound to the satellite as a function of time for orbit 2. Dotted, red solid and blue-dashed curves are used to indicate the dark matter, and stellar components $C_{1}$ and $C_{2}$ respectively. In the bottom the evolution with time of the ratio between the velocity dispersions of $C_{1}$ and $C_{2}$ is shown for two regions within the dwarf: stars within two core radii (magenta dashed) and stars approximately lying closer to the tidal radius (green solid). These ratios have been normalized to the original values $\left(\Delta_{\sigma_{1,2}}^{0}\right.$, thin black line in the right panel).

density profiles. For example, the model in orbit 4 shows an increase in $\sigma_{\text {los }}$ at $R \sim 600 \mathrm{pc}$, where the fraction of unbound stars accounts for $\sim 8-10 \%$ of the mass at that radius. However, bottom right panel of Figure 4 shows that an excess of stars appears in the surface density only for $R>1500 \mathrm{pc}$, when the unbound contribution is $\geq 20 \%$.

We finally point out that the shape of the line-of-sight velocity profiles may depend on the level of rotational support of the initial progenitor, which we have neglected in this study. A comparison between properties of the remnant of a disky versus a spheroidal progenitor is undoubtedly interesting and deferred to future work.

The preferential pruning by tides of the more extended component is the main responsible for the attenuation of the kinematical distinction between both stellar populations. This can be seen on the Figure 6(a), which shows the final velocity gap between components $C_{1}$ and $C_{2}$ as a function of distance from the center of the satellite. The vertical axis corresponds to the ratio between the velocity dispersion in these components, $\Delta_{\sigma_{1,2}}=\sigma_{C_{1}} / \sigma_{C_{2}}$, for each of the orbits we have explored. For an easier comparison, the black dotted curve shows the initial configuration. When the model satellite is evolved on orbits such as 1 or 3 (solid magenta, long-dashed red) the distinction set by construction between both luminous populations remains essentially unchanged at any radius within the dwarf. In spite of the pruning of $60-80 \%$ of the dark matter content on these satellites, these two experiments showed negligible stripping of their stellar components, signaling that a certain degree of stellar mass removal must take place before the relative kinematics of the two populations is noticeably altered.

On the other hand, the simulated satellites on orbits 2 and 4 experienced significant stellar stripping and show kinematical gaps between the luminous components that have been reduced by $\sim 20-30 \%$. Such "mixing" proceeds typically outside-in, starting soon after the tides begin to strip stars from the dwarf. Figure 6(b) shows the evolution of the bound mass with time (upper panel) together with the kinematical gap (bottom panel) for the simulated satellite on orbit 2. Notice that mass removal is not a continuous process but rather presents a stepwise behaviour closely related to the orbital pericenter crossings. After the first 


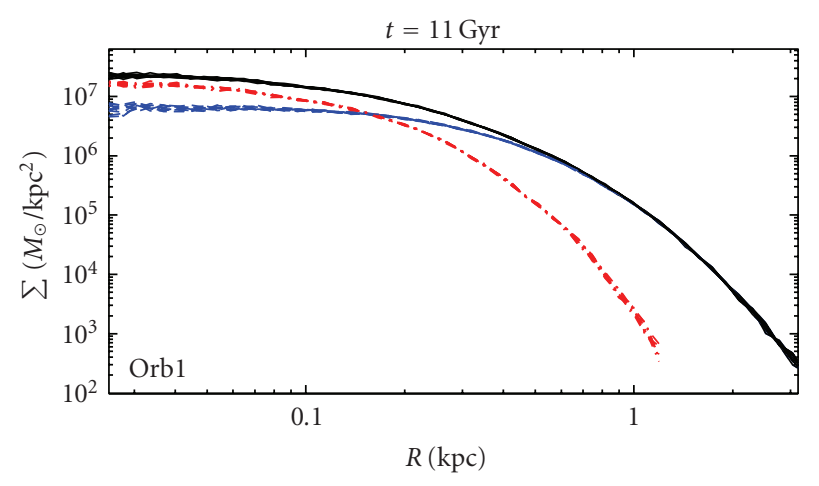

(a)

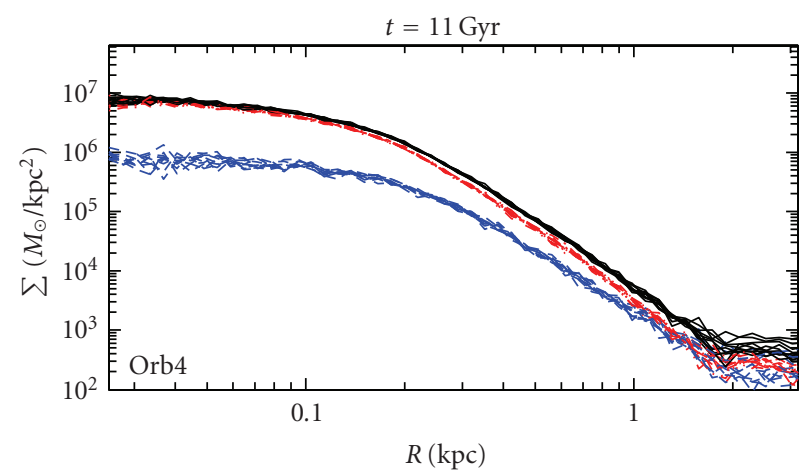

(c)

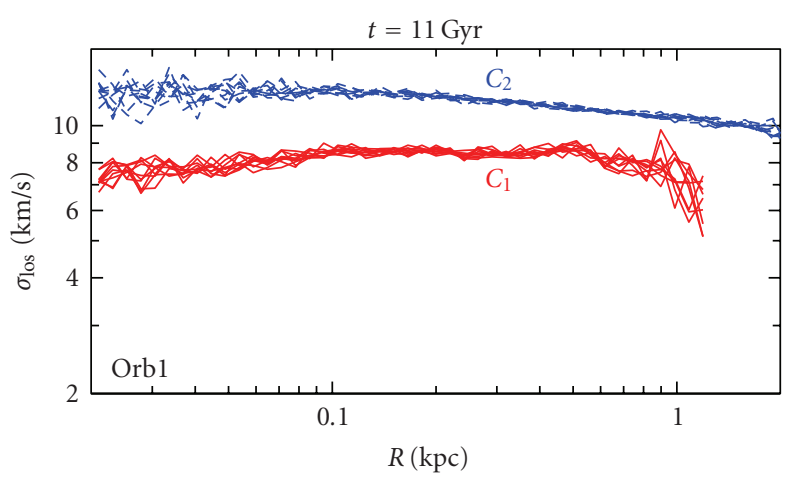

(b)

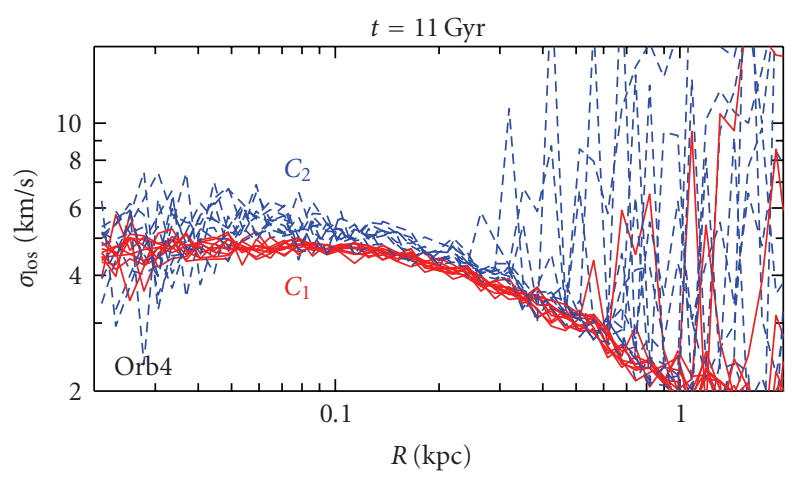

(d)

Figure 7: Projected density (a) and velocity dispersion (b) profiles for satellites evolved in Orb1 and Orb4 for a longer time interval: $t \sim 11 \mathrm{Gyr}$, instead of the fiducial $t \sim 6 \mathrm{Gyr}$. Notice for Orb4 that, as mass depletion proceeds further, the spatial and kinematical segregation between $C_{1}$ and $C_{2}$ is increasingly erased.

pericenter passage, the dark matter content of the satellite has dropped by almost a factor of 2 . However, the stellar mass is more resilient and shows signs of ongoing stripping only after the third pericentric passage, when the dark matter mass has shrunk to only $\sim 10 \%$ of the original value. It is thus when the stripping of the stellar mass begins that the relative kinematical differences between the two luminous components show a significant departure from the original configuration (see bottom right panel of Figure 6). This figure also shows that only the more extended $\left(C_{2}\right)$ stellar component is affected, with $C_{1}$ conserving $100 \%$ of its initial mass during the whole time integration. This helps to explain why the kinematical gap changes more appreciably in the outskirts than in the core of the satellite galaxy, which will tend to remain less affected by tidal forces.

3.3. Effect of a Different Time Integration. Models that attempt to reproduce the present-day properties of dwarf galaxies are usually degenerate with respect to the unknown initial structure of the objects (density profile, mass, characteristic scales) and their orbital paths. Even though current data allow to reasonably characterize the present-day structural properties of the nearby dwarf galaxies, these are a combined result of (i) their initial configuration, (ii) their orbital motion within the Local Group, and (iii) evolution of the host gravitational potential field, all three factors with hardly any strong observational constraints. Proper motions help us to partially reconstruct the orbits, but unfortunately, errors are still prohibitive, with uncertainties of the order $\sim 20-100 \%[28,29,41]$.

As a rule of thumb, for a fixed final configuration, more massive, concentrated objects are needed if we desire to increase the time integration in the host potential keeping the orbit of the object fixed (see, e.g., [42, Section 3.3]). For the analysis presented above, we have chosen a fiducial value for the time integration of $t \sim 6 \mathrm{Gyr}$; which is, at some extent, arbitrary. We explore in Figure 7 the effects induced by a longer time integration $(t \sim 11 \mathrm{Gyr})$ on the density profile (a) and velocity dispersion (b) for two of our models (Orb1 and Orb4).

For external orbits such as Orb1, with long orbital period and large pericenter distances, the effect of (almost) doubling the time integration is negligible. Objects on such orbits can preserve most of their luminous component bound, showing very little evolution in their spatial and kinematical properties with respect to the initial configuration.

On the other hand, if tides are as strong as on Orb4, the increase in the integration translates into lower densities in the core of the dwarf, and to a further mixing of the spatial and kinematical profiles of both stellar populations. For example, for the satellite evolved in Orb4 during $t \sim$ $11 \mathrm{Gyr}$, the bound mass fraction of components $C_{1}$ and $C_{2}$ drop to about $38 \%$ and $4 \%$ of their initial configuration, 
respectively. This seems to be enough to completely erase the kinematical gap between both components (see Figure 7(d)) as well as their initial spatial segregation (Figure $7(\mathrm{c})$ ).

We note that this object shows, at the end of the experiment, an overall velocity dispersion $\sigma \sim 5 \mathrm{~km} / \mathrm{s}$, that is about half the initial value, quite low compared to the typical value of the classical satellites in the Local Group. However, given the degeneracies in this type of modelling mentioned above, a larger velocity dispersion $\sigma_{\text {los }} \sim 8-9 \mathrm{~km} / \mathrm{s}$ may be reconciled with a time integration as long as $t \sim$ $11 \mathrm{Gyr}$ provided we increase accordingly the initial $\sigma_{\text {los }}$ of the progenitor system [42].

The behaviour shown in Figure 7 confirms the conclusions from Section 3; namely, that tides affecting the luminous components are needed in order to effectively erase the structural and kinematical biases between two initially segregated stellar populations.

\section{Application to the Local Group: Sculptor and Carina Dwarfs}

The results reported in Section 3 can be extrapolated to particular examples within the Local Group. Among the dwarf spheroidals orbiting around our Galaxy, Carina and Sculptor are perhaps the most instructive cases to interpret in light of our results given their proximity and good quality data currently available. Sculptor shows clear spatial variations in the properties of its stellar populations, such as spatial variations in the relative distribution of RHB and BHB stars, a clear metallicity variation with radius as derived from spectroscopic data and also kinematical differences associated to each metallicity population. Carina instead shows no gradient in the distribution of horizontal branch stars themselves, but a distinction is possible between $\mathrm{HB}$ stars and red clump stars. Signs of a weak metallicity gradient were found by Koch et al. [11] using $\sim 500$ giant branch stars. However this gradient is only mild and, unlike Sculptor, no kinematical distinction has been identified so far.

The Sculptor and Carina dSphs are found today at comparable distances from the Milky Way $(\sim 100 \mathrm{kpc})$, although numerical integration of their proper motions suggests that their orbital paths could have been quite different in the past (see Orb1 and Orb2 in Figure 2). The most likely orbit of Carina seems to be confined much more to the inner regions of the Milky Way halo, with smaller pericenter passages and shorter periods than that of Sculptor. Our numerical experiments suggest that a satellite galaxy orbiting in a Carina-like orbit (Orb2) is likely to be exposed to tidal forces that drive significant mass removal. This in turn triggers a mixing and weakening of the kinematical gap that may have existed between its luminous components, which may be challenging to detect with the current spectroscopic samples.

On the other hand, the same model evolved in a more external orbit like Sculptor's maintains the kinematical and spatial properties of the luminous components almost unchanged after $\sim 6$ Gyr of evolution in the host potential.

A simple extrapolation of these results suggests that the lack of a stronger and clear metallicity gradient in Carina, or equivalently, the nondetection of distinct kinematics associated to different stellar populations might be partly due to the effect of strong tidal forces inherent to Carina's orbit. It is possible that in the past, Carina could have had a double component configuration, where the metallicity gradient and kinematical gaps were larger than today's measurements suggest. Notice that according to our simulations, this can only happen if tidal stripping has already taken place on the luminous components of Carina. Interestingly, observational evidence points toward the presence of unbound stars beyond the formal tidal radius of this dwarf [43, 44], (however see discussion in [45]).

These conclusions are subject to an important caveat. Our models do not take into account the gas content of the satellite, and therefore, all star formation activity that might occur after the infall time (our fiducial $t \sim 6 \mathrm{Gyr}$ ) has been neglected. This could be of particular relevance for Carina, given the more extended star formation activity suggested by observations (e.g., Rizzi et al. [46]). Simulations have shown that during pericenter passages episodes of star formation may be triggered for gas rich systems (Mayer et al. $[14,47]$ ). Such events would reestablish a spatial (and presumably kinematical) bias between the old and newly formed stars. This could certainly lower the efficiency of tides to spatially and kinematically mix the dwarf's stellar populations discussed in our models. This is, however, unlikely to render invalid the results explored in Section 3, and their applicability to Carina. Notice that according to the recent models of Carina's star formation history, the bulk of stars is older than $\sim 5 \mathrm{Gyr}[46,48]$, which means that subsequent star formation episodes are unlikely to contribute to a large fraction of the total mass. Moreover, after the satellite has lost most of its dark matter mass, the mixing proceeds fast as the luminous components start to become stripped. Therefore, depending on the orbit it is still possible that mixing could have worked, at least partially, in Carina even if modest integration times, comparable or lower than the age of the dominant population, are invoked.

All these arguments are based on a model of a dwarf galaxy with two initially segregated stellar components. However, neither the origin nor how often galaxies are expected to show such configurations has yet been properly understood. Further constraints on the validity (or not) of the extrapolations of our results to cases such as that of Carina's will come from a better understanding of the mechanisms able to generate metallicity gradients and spatial plus kinematical segregation of stellar populations in the dwarf spheroidal galaxies.

\section{Discussion and Conclusions}

We have studied, by means of N-body numerical simulations, the effects of the tidal forces induced by a host galactic potential on a multicomponent satellite galaxy. The model satellite is set up adhoc to initially have two spherical different stellar populations, that are kinematically and spatially segregated. A more centrally concentrated and with lower velocity dispersion $C_{1}$ (reminiscent of a "metal rich" 
population) and a more extended, higher velocity ("metal poor") $C_{2}$ population. These two components are deeply embedded within a dark matter halo that largely dominates the total mass of the system. This satellite model roughly matches the structural properties of classical Local Group dwarfs. We have followed the evolution of such object in time for $t \sim 6$ Gyr on four different orbits around a Milky Waylike host.

We find that the ability to distinguish kinematically the two stellar components (set initially in our model to differ by $\sigma_{2}-\sigma_{1} \sim 4 \mathrm{~km} / \mathrm{s}$ ) is strongly dependent on the amplitude of the tides experienced by the satellite during its orbit. In cases where a significant amount of mass has been removed, the velocity gap between the more concentrated (colder) stellar population and the more extended (hotter) component can decrease between $\sim 30-70 \%$ of its initial value, thus partly erasing the initial kinematical segregation between the stellar populations. The magnitude of this effect is tightly related to the tidal stripping experienced by the satellite, and in particular, the removal of luminous mass is necessary for this effect to be significant. Such conditions are more easily obtained for orbits restricted to the inner regions of the host potential, whose close pericenter passages and short orbital periods promote the tidal stripping of the satellite's particles.

We apply these ideas to Sculptor and Carina, two of the classical dwarf galaxies of the Milky Way. Sculptor shows a stronger metallicity gradient, together with a lower velocity dispersion for the more centrally concentrated, metal rich stars compared to the metal poor population. On the other hand, in Carina trends are considerably more subtle, with no kinematical distinction between populations detected to date. We argue that differences in the orbital paths of these two dwarfs can be partially responsible for the weakening of the velocity gap in Carina, while leaving Sculptor unaffected due to its more external and longer period orbit.

Further validation of the tidal "mixing" scenario that has been proposed in this Paper depends upon efforts from theoretical as well as observational studies. Comprehensive models to understand the formation of the metallicity gradients as well as their likelihood in dwarf galaxies are of fundamental relevance. The structure of the dark matter halos of dwarf spheroidals (core vs cusped) might also play an important role. Cored profiles could provide less gravitational support to oppose the tidal stripping forces compared to our cusped models, perhaps changing (albeit we expect only quantitatively) the timescales and relevance of the effects studied here. Larger samples of line-of-sight velocities for stars associated to dwarfs spheroidals of the Local Group may improve the detectability of double stellar components kinematically segregated in cases where such feature has not yet been identified. Finally, our models predict that some degree of mass depletion on the stellar components must take place in order to considerably affect the kinematical segregation of dwarfs with composite stellar populations. Deep photometric surveys mapping the outskirts of these satellites, specially beyond their tidal radii, should be able to provide definite clues on the existence (or not) of tidally unbound stars associated to each of these objects, a necessary (although not sufficient) condition for our models to apply.

\section{Acknowledgments}

The first author would like to thank Eline Tolstoy for enlightening discussions. The first author and The second author gratefully acknowledge NWO and NOVA and the Coimbra Group for financial support. They also thank both anonymous referees for useful suggestions and comments.

\section{References}

[1] M. G. Walker, M. Mateo, E. W. Olszewski, et al., "Velocity dispersion profiles of seven dwarf spheroidal galaxies," The Astrophysical Journal, vol. 667, no. 1, pp. L53-L56, 2007.

[2] L. E. Strigari, J. S. Bullock, M. Kaplinghat, et al., "A common mass scale for satellite galaxies of the Milky Way," Nature, vol. 454, no. 7208, pp. 1096-1097, 2008.

[3] J. S. Gallagher III and R. F. G. Wyse, "Dwarf spheroidal galaxies: keystones of galaxy evolution," Astronomical Society of the Pacific, vol. 106, pp. 1225-1238, 1994.

[4] M. Mateo, "Dwarf galaxies of the Local Group," Annual Review of Astronomy \& Astrophysics, vol. 36, no. 1, pp. 435-506, 1998.

[5] E. K. Grebel, J. S. Gallagher III, and D. Harbeck, "The progenitors of dwarf spheroidal galaxies," The Astronomical Journal, vol. 125, no. 4, pp. 1926-1939, 2003.

[6] D. Harbeck, E. K. Grebel, J. Holtzman, et al., "Population gradients in local group dwarf spheroidal galaxies," The Astronomical Journal, vol. 122, no. 6, pp. 3092-3105, 2001.

[7] E. Tolstoy, M. J. Irwin, A. Helmi, et al., "Two distinct ancient components in the sculptor dwarf spheroidal galaxy: first results from the dwarf abundances and radial velocities team," The Astrophysical Journal, vol. 617, no. 2, pp. L119-L122, 2004.

[8] D. Faria, S. Feltzing, I. Lundström, et al., "The usage of Strömgren photometry in studies of local group dwarf spheroidal galaxies: application to Draco: a new catalogue of Draco members and a study of the metallicity distribution function and radial gradients," Astronomy \& Astrophysics, vol. 465, no. 2, pp. 357-373, 2007.

[9] A. W. McConnachie, N. Arimoto, and M. Irwin, "Deconstructing dwarf galaxies: a suprime-cam survey of Andromeda II," Monthly Notices of the Royal Astronomical Society, vol. 379, no. 1, pp. 379-392, 2007.

[10] A. Koch, M. I. Wilkinson, J. T. Kleyna, et al., "Stellar kinematics and metallicities in the Leo I dwarf spheroidal galaxy-wide-field implications for galactic evolution," The Astrophysical Journal, vol. 657, no. 1, article 1, pp. 241-261, 2007.

[11] A. Koch, E. K. Grebel, R. F. G. Wyse, et al., "Complexity on small scales: the metallicity distribution of the Carina dwarf spheroidal galaxy," The Astronomical Journal, vol. 131, no. 2, pp. 895-911, 2006.

[12] G. Battaglia, E. Tolstoy, A. Helmi, et al., "The DART imaging and CaT survey of the Fornax dwarf spheroidal galaxy," Astronomy \& Astrophysics, vol. 459, no. 2, pp. 423-440, 2006.

[13] D. Kawata, N. Arimoto, R. Cen, and B. K. Gibson, "Origin of two distinct populations in dwarf spheroidal galaxies," The Astrophysical Journal, vol. 641, no. 2, pp. 785-794, 2006.

[14] L. Mayer, F. Governato, M. Colpi, et al., "Tidal stirring and the origin of dwarf spheroidals in the local group," The Astrophysical Journal, vol. 547, no. 2, pp. L123-L127, 2001.

[15] L. Mayer, F. Governato, M. Colpi, et al., "The metamorphosis of tidally stirred dwarf galaxies," The Astrophysical Journal, vol. 559, no. 2, pp. 754-784, 2001. 
[16] V. Springel, S. D. M. White, A. Jenkins, et al., "Simulations of the formation, evolution and clustering of galaxies and quasars," Nature, vol. 435, no. 7042, pp. 629-636, 2005.

[17] J. F. Navarro, C. S. Frenk, and S. D. M. White, "A universal density profile from hierarchical clustering," The Astrophysical Journal, vol. 490, no. 2, pp. 493-508, 1997.

[18] A. Klypin, H. Zhao, and R. S. Somerville, " $\Lambda$ CDM-based models for the Milky Way and M31. I. Dynamical models," The Astrophysical Journal, vol. 573, no. 2, pp. 597-613, 2002.

[19] L. Hernquist, "An analytical model for spherical galaxies and bulges," The Astrophysical Journal, vol. 356, no. 2, pp. 359-364, 1990.

[20] M. Miyamoto and R. Nagai, "Three-dimensional models for the distribution of mass in galaxies," Publications of the Astronomical Society of Japan, vol. 27, pp. 533-543, 1975.

[21] K. V. Johnston, S. Sigurdsson, and L. Hernquist, "Measuring mass-loss rates from galactic satellites," Monthly Notices of the Royal Astronomical Society, vol. 302, no. 4, pp. 771-789, 1999.

[22] L. Hernquist, "N-body realizations of compound galaxies," The Astrophysical Journal, vol. 86, no. 2, pp. 389-400, 1993.

[23] E. Athanassoula, E. Fady, J. C. Lambert, and A. Bosma, "Optimal softening for force calculations in collisionless $\mathrm{N}$ body simulations," Monthly Notices of the Royal Astronomical Society, vol. 314, no. 3, pp. 475-488, 2000.

[24] A. W. McConnachie, J. Peñarrubia, and J. F. Navarro, "Multiple dynamical components in local group dwarf spheroidals," Monthly Notices of the Royal Astronomical Society, vol. 380, no. 1, pp. L75-L79, 2007.

[25] E. L. Łokas, "Velocity dispersions of dwarf spheroidal galaxies: dark matter versus MOND," Monthly Notices of the Royal Astronomical Society, vol. 327, no. 2, pp. L21-L26, 2001.

[26] E. Hayashi, J. F. Navarro, J. E. Taylor, J. Stadel, and T. Quinn, "The structural evolution of substructure," The Astrophysical Journal, vol. 584, no. 2, pp. 541-558, 2003.

[27] J. Peñarrubia, A. W. McConnachie, and J. F. Navarro, "The cold dark matter halos of local group dwarf spheroidals," The Astrophysical Journal, vol. 672, no. 2, pp. 904-913, 2008.

[28] S. Piatek, C. Pryor, E. W. Olszewski, et al., "Proper motions of dwarf spheroidal galaxies from Hubble space telescope imaging. II. Measurement for Carina," The Astronomical Journal, vol. 126, no. 5, pp. 2346-2361, 2003.

[29] S. Piatek, C. Pryor, P. Bristow, et al., "Proper motions of dwarf spheroidal galaxies from Hubble Space Telescope imaging. IV. Measurement for sculptor," The Astronomical Journal, vol. 131, no. 3, pp. 1445-1460, 2006.

[30] J. S. Bullock and K. V. Johnston, "Tracing galaxy formation with stellar halos. I. Methods," The Astrophysical Journal, vol. 635, no. 2, pp. 931-949, 2005.

[31] A. S. Font, K. V. Johnston, J. S. Bullock, and B. E. Robertson, "Chemical abundance distributions of galactic halos and their satellite systems in a $\Lambda \mathrm{CDM}$ universe," The Astrophysical Journal, vol. 638, no. 2, pp. 585-595, 2006.

[32] A. S. Font, K. V. Johnston, J. S. Bullock, and B. E. Robertson, "Phase-space distributions of chemical abundances in Milky Way-type galaxy halos," Astrophysical Journal, vol. 646, no. 2, pp. 886-898, 2006.

[33] L. V. Sales, J. F. Navarro, M. G. Abadi, and M. Steinmetz, "Satellites of simulated galaxies: survival, merging and their relationto the dark and stellar haloes," Monthly Notices of the Royal Astronomical Society, vol. 379, no. 4, pp. 1464-1474, 2007.

[34] J. Peñarrubia, J. F. Navarro, and A. W. McConnachie, "The tidal evolution of local group dwarf spheroidals," The Astrophysical Journal, vol. 673, no. 1, pp. 226-240, 2008.
[35] J. Klimentowski, E. L. Łokas, S. Kazantzidis, F. Prada, L. Mayer, and G. A. Mamon, "Mass modelling of dwarf spheroidal galaxies: the effect of unbound stars from tidal tails and the Milky Way," Monthly Notices of the Royal Astronomical Society, vol. 378, no. 1, pp. 353-368, 2007.

[36] G. Battaglia, A. Helmi, E. Tolstoy, M. Irwin, V. Hill, and P. Jablonka, "The kinematic status and mass content of the sculptor dwarf spheroidal galaxy," The Astrophysical Journal, vol. 681, no. 1, pp. L13-L16, 2008.

[37] A. Helmi, J. F. Navarro, B. Nordström, J. Holmberg, M. G. Abadi, and M. Steinmetz, "Pieces of the puzzle: ancient substructure in the Galactic disc," Monthly Notices of the Royal Astronomical Society, vol. 365, no. 4, pp. 1309-1323, 2006.

[38] J. Binney and S. Tremaine, Galactic Dynamics, Princeton University Press, Princeton, NJ, USA, 2nd edition, 2008.

[39] U. Ural and M. Wilkinson, "Multiple stellar populations in dwarf spheroidal galaxies," Astronomische Nachrichten, vol. 329, no. 9-10, pp. 1040-1041, 2008.

[40] M. Bellazzini, H. J. Newberg, M. Correnti, F. R. Ferraro, and L. Monaco, "Detection of a population gradient in the Sagittarius stream," Astronomy \& Astrophysics, vol. 457, no. 2, pp. L21L24, 2006.

[41] M. G. Walker, M. Mateo, and E. W. Olszewski, "Systemic proper motions of milky way satellites from stellar redshifts: the Carina, Fornax, Sculptor, and Sextans dwarf spheroidals," The Astrophysical Journal, vol. 687, no. 2, part 2, pp. L75-L78, 2008.

[42] L. V. Sales, A. Helmi, E. Starkenburg, et al., "On the genealogy of the Orphan Stream," Monthly Notices of the Royal Astronomical Society, vol. 389, no. 3, pp. 1391-1398, 2008.

[43] S. R. Majewski, P. M. Frinchaboy, W. E. Kunkel, et al., "Exploring halo substructure with giant stars. VI. Extended distributions of giant stars around the Carina dwarf spheroidal galaxy: how reliable are they?" The Astronomical Journal, vol. 130, no. 6, pp. 2677-2700, 2005.

[44] R. R. Muñoz, S. R. Majewski, S. Zaggia, et al., "Exploring halo substructure with giant stars. XI. The tidal tails of the Carina dwarf spheroidal galaxy and the discovery of magellanic cloud stars in the Carina foreground," The Astrophysical Journal, vol. 649, no. 1, pp. 201-223, 2006.

[45] J. Peñarrubia, J. F. Navarro, A. W. McConnachie, and N. F. Martin, "The signature of galactic tides in local group dwarf spheroidals," The Astrophysical Journal, vol. 698, no. 1, pp. 222-232, 2009.

[46] L. Rizzi, E. V. Held, G. Bertelli, and I. Saviane, "Clues to the evolution of the Carina dwarf spheroidal galaxy from the color distribution of its red giant stars," The Astrophysical Journal, vol. 589, no. 2, pp. L85-L88, 2003.

[47] L. Mayer, C. Mastropietro, J. Wadsley, J. Stadel, and B. Moore, "Simultaneous ram pressure and tidal stripping; how dwarf spheroidals lost their gas," Monthly Notices of the Royal Astronomical Society, vol. 369, no. 3, pp. 1021-1038, 2006.

[48] D. Hurley-Keller, M. Mateo, and J. Nemec, "The star formation history of the Carina dwarf galaxy," The Astronomical Journal, vol. 115, no. 5, pp. 1840-1855, 1998. 

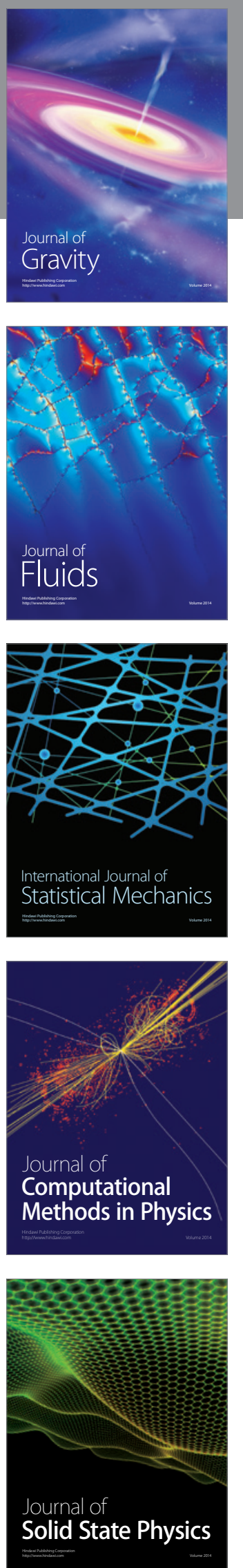

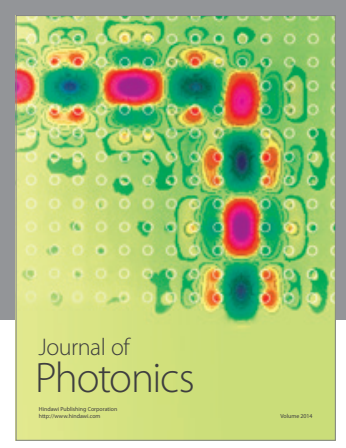

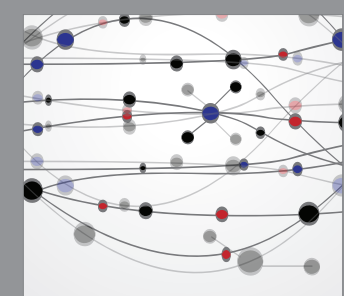

The Scientific World Journal
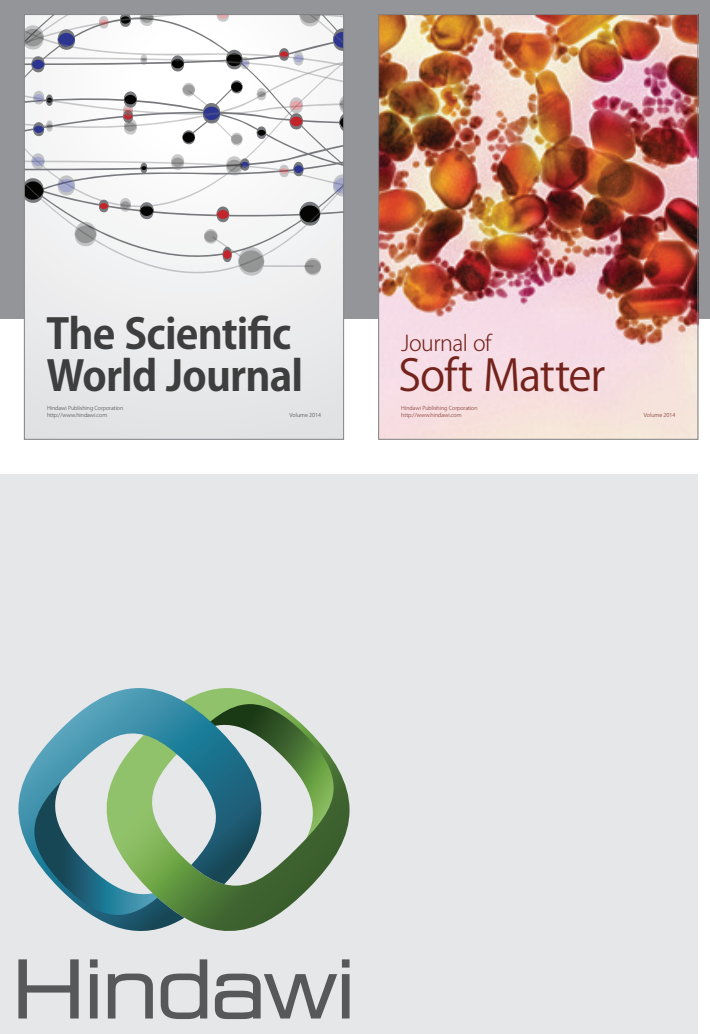

Submit your manuscripts at

http://www.hindawi.com
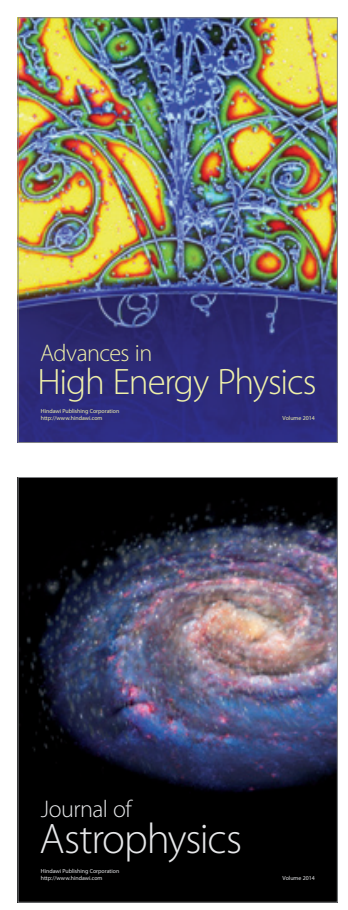
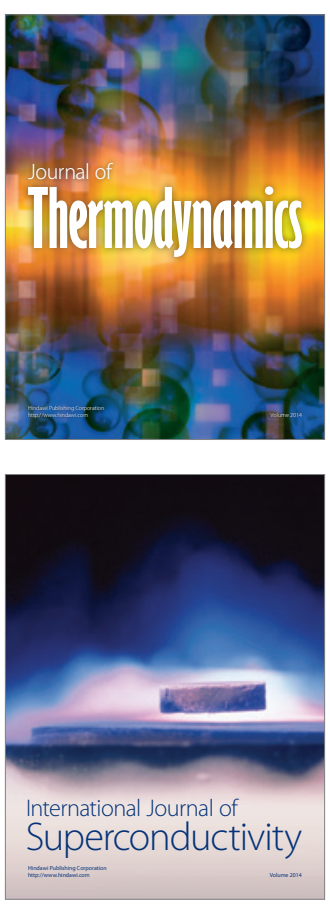
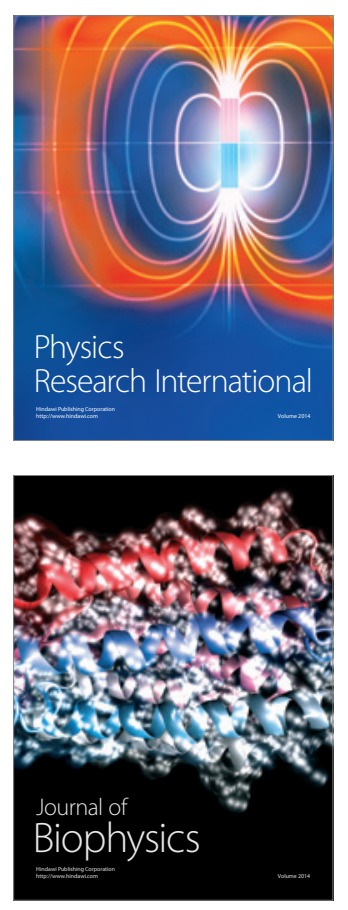
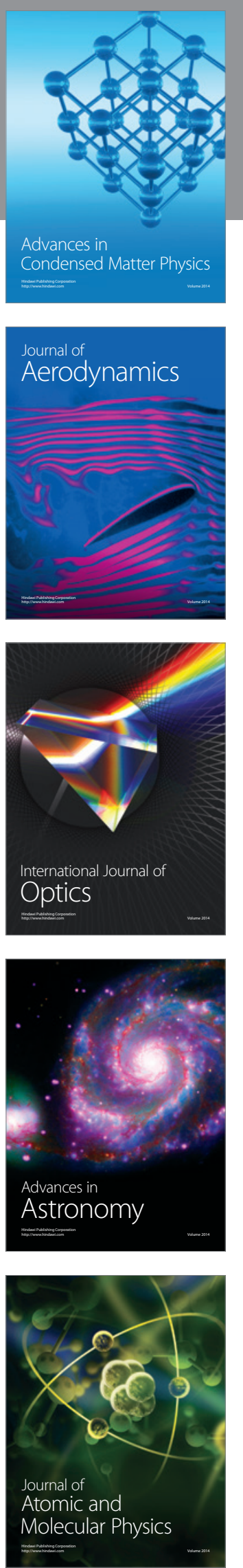